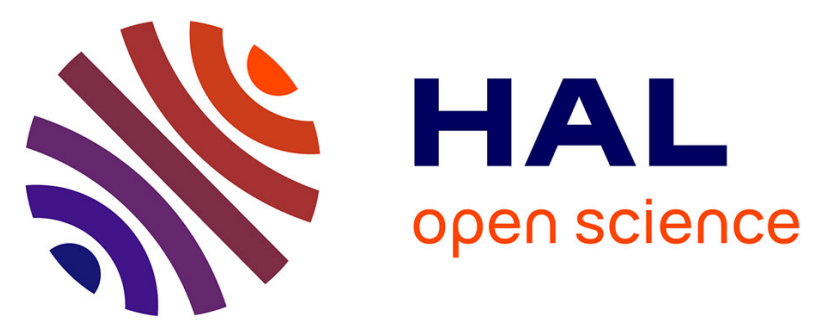

\title{
Segregated versus Disordered Stacking in Two Low Bandgap Alternated Copolymers for Photovoltaic Applications: Impact of Polymorphism on Optical Properties
}

Yuhan Zhong, Laure Biniek, Nicolas Leclerc, Stéphanie Ferry, Martin

Brinkmann

\section{To cite this version:}

Yuhan Zhong, Laure Biniek, Nicolas Leclerc, Stéphanie Ferry, Martin Brinkmann. Segregated versus Disordered Stacking in Two Low Bandgap Alternated Copolymers for Photovoltaic Applications: Impact of Polymorphism on Optical Properties. Macromolecules, 2018, 51 (11), pp.4238-4249. 10.1021/acs.macromol.8b00378 . hal-02338356

\section{HAL Id: hal-02338356 https://hal.science/hal-02338356}

Submitted on 7 Dec 2021

HAL is a multi-disciplinary open access archive for the deposit and dissemination of scientific research documents, whether they are published or not. The documents may come from teaching and research institutions in France or abroad, or from public or private research centers.
L'archive ouverte pluridisciplinaire HAL, est destinée au dépôt et à la diffusion de documents scientifiques de niveau recherche, publiés ou non, émanant des établissements d'enseignement et de recherche français ou étrangers, des laboratoires publics ou privés. 


\section{Segregated versus disordered stacking in two low bandgap alternated copolymers for photovoltaic applications : Impact of polymorphism on optical properties}

Yuhan Zhong ${ }^{1}$, Laure Biniek ${ }^{1}$, Nicolas Leclerc ${ }^{2}$, Stéphanie Ferry ${ }^{2}$ and Martin Brinkmann $^{1}$

(1) Institut Charles Sadron, CNRS-Université de Strasbourg, 23 rue du Loess, Strasbourg 67034, France

(2) Institut de Chimie et Procédés pour l'Energie, l'Environnement et la Santé, UMR 7515, ECPM, 25 rue Becquerel, 67087 Strasbourg Cedex2, France 
Figure for Table of Content
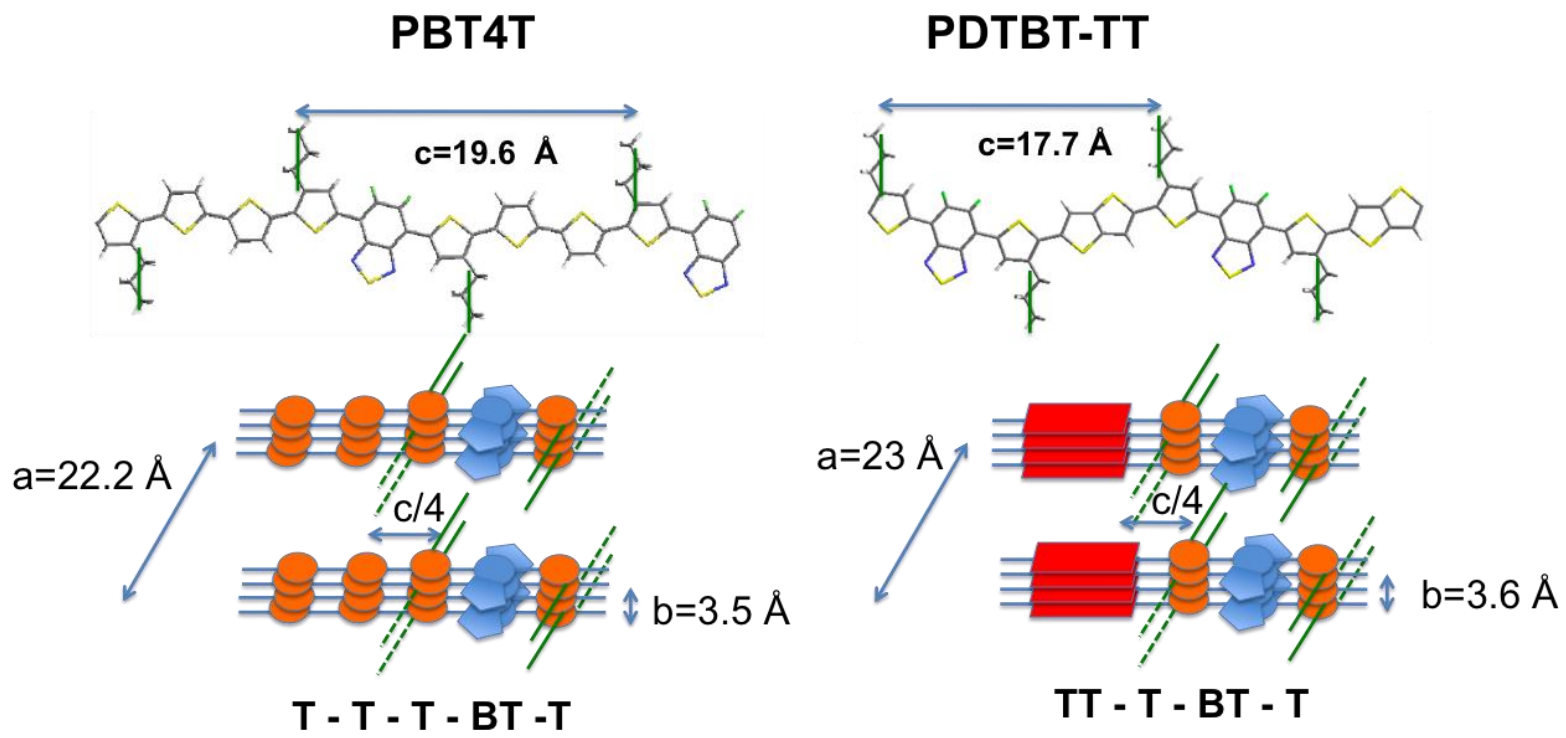


\begin{abstract}
Structure-property correlations are often hampered by insufficient structural insight in the crystal packing of polymer semiconductors widely used in electronic devices such as organic solar cells. Herein, both the semi-crystalline morphology and the crystalline structures of two high performance polymer semiconductors showing more than $9 \%$ efficencies, namely PDTBT-TT and PBT4T, are established by a combination of oriented crystallization and transmission electron microscopy. PDTBT-TT and PBT4T form layered structures with alternation of $\pi$-stacked backbones and layers of disordered alkyl side chains. $\pi$-stacking is such that benzothiadiazol and the co-monomer (quaterthiophene or thienothiophene-bithiophene) segregate to form distinct stacks. This segregated stacking is preferentially obtained in thin films aligned by high temperature rubbing at $\mathrm{T}=200^{\circ} \mathrm{C}-230^{\circ} \mathrm{C}$. However, the two polymers show different stabilities of this polymorph versus temperature. The segregated stacking of PDTBT-TT is stable up to near the melting temperature whereas for PBT4T, it transforms to a layered structure with significant intra-stack disorder at $\mathrm{T} \geq 250^{\circ} \mathrm{C}$. The intensity of the 0 0 component of the vibronic progression in the absorption spectrum is enhanced for the polymorph with long-range segregated $\pi$-stacks. The structural models determined for the two polymers suggest that both the position of alkyl side chains and the preferential $\pi$-stacking interactions between co-monomers determine the polymorphism and corresponding thermal stability.
\end{abstract}

Corresp. author : martin.brinkmann@ics-cnrs.unistra.fr 


\section{Introduction.}

Alternating semiconducting copolymers made of electron-rich and electron-poor units in the backbone have attracted much interest due to the possibility to tune their electronic properties by an adequate combination of donor and acceptor comonomers. $^{1-4}$ This strategy has been particularily fruitful to control opto-electronic properties of the active layers in organic photovoltaic cells. However, minute modifications in the chemical structure of the polymers (the side chain position or the branched/ramified nature of side chains) impact strongly the resulting optoelectronic properties in thin film devices. ${ }^{5}$ The alkyl side chain structure is known to influence the orientation of the crystals on the substrate as well as the type of stacking of the chains in the crystals. From a structural standpoint, the periodic alternation of donor and acceptor units in the chains implies different possibilities of $\pi$-stacking, the extreme cases corresponding to segregated and to fully mixed stacking.6,7 As reported recently for Poly(9,9-di-n-cyclopentadithiophene-alt-benzothiadiazole) (PCPDTBT) and earlier for Poly[N,N'-bis(2-octyldodecyl)-1,4,5,8-naphthalene-dicarboximide-2,6-diyl]-alt-5,5'(2,2'-bithiophene) (P(NDI2OD-T2)), different polymorphs corresponding to these two stacking modes were identified and their impact on optical and device properties established. $6,7,8$

Due to enhanced aggregation in solution, many recent low bandgap polymer semiconductors (PSCs), especially fluorinated polymers, ${ }^{9}$ exhibit intense low energy aggregate bands in the range $700-800 \mathrm{~nm}$ in their UV-vis absorption spectra. ${ }^{9-12}$ The exact structural origin of these optical features remains unknown simply because the packing of the chains in the corresponding polymer crystals or aggregates in solution could not be precisely addressed. Only in some polymers such as p(NDI20DT2) was it 
possible to draw some correlation between the packing of the chains and the presence of this aggregate band. ${ }^{6}$ This optical feature is present in the polymorph that shows segregated stacking of naphthalene diimide (NDI) and bithiophene (T2) units whereas it is strongly reduced in intensity in the polymorph (form II) with mixed stacking of NDI and T2. ${ }^{6}$ Is the correlation between the segregated/mixed stacking of the comonomers and the aggregate-band in UV-vis spectra a general phenomenon in alternated conjugated copolymers? To address this question, it is necessary to investigate the packing of the polymer chains in the crystals. This implies a precise structural control in thin films, which is usually far from being observed in spin-coated or doctor-bladed thin films.

Several methods allow to grow PSCs thin films with high crystalline perfection. Solvent vapor annealing has been used to grow single-crystal like systems in P3HT (form II) and in PCPDTBT, and it helped identify the packing of the chains in the stable thermodynamic polymorphs. ${ }^{13-16}$ Other approaches to induce high crystallinity and orientation were proposed such as zone casting, ${ }^{17}$ epitaxy ${ }^{18-21}$ or high-temperature rubbing (HTR). ${ }^{22-27}$ It has been demonstrated that HTR is an effective method to prepare highly oriented thin films with a precise control of both semi-crystalline morphology and crystalline perfection. ${ }^{25,27}$ In regioregular poly(3-hexylthiophene) (P3HT) for instance, the lamellar periodicity and crystal dimensions in the chain direction are controlled by adjusting the rubbing temperature $\left(\mathrm{T}_{\mathrm{R}}\right)$. For $\mathrm{T}_{\mathrm{R}}=230^{\circ} \mathrm{C}$, highly oriented and crystalline P3HT films were obtained (60\% crystallinity). Correlations between the length of planarized chain segments $l_{c}$ and the excitonic bandwidth W were established: the larger $l_{c}$, the lower W. ${ }^{27}$ Similarly, in PCPDTBT and F-PCPDTBT, the structure of the polymers was refined with the help of well defined fiber-like patterns obtained by electron diffraction on the films oriented by HTR. ${ }^{16}$ 
In this contribution, the structure and morphology in highly oriented thin films is correlated with the optical features in the UV-vis absorption spectra for two polymer semiconductors widely used in highly efficient photovoltaic solar cells, namely Poly[(5,6-difluoro-2,1,3-benzothiadiazol-4,7-diyl)-alt-(3,3"')-di(2-octyldodecyl)-

2,2';5',2";5",2'"'-quaterthiophen-5,5'"'-diyl)] (PBT4T hereafter, also known as PCE11) and (PDTBT-TT). Both polymers yield power conversion efficencies close to $10 \%$ in single junction devices. ${ }^{28,29}$ The two polymers share a common 4,7-bis(4-(2octyldodecyl)thiophen-2-yl)-5,6-difluorobenzo[c][1,2,5]thiadiazole unit including the electron deficient benzothiadiazole moiety, but differ in the co-monomer structure. The comonomer is a bithiophene in PBT4T and a more rigid thieno[3,2-b]thiophene (TT) in PDTBT-TT. It has been demonstrated that PDTBT-TT shows stronger interchain interactions in solution than PBT4T and that the differences in Differential Scanning Calorimetry and X-Ray diffraction characteristics arise from the more rigid TT monomer. ${ }^{28}$ However, previous studies could not determine the type of stacking (segregated versus mixed) in the crystal phases of both these polymers.

This study presents a systematic analysis of the correlations existing between the structure, analyzed by Transmission Electron Microscopy (TEM), and the corresponding optical absorption properties in oriented films of PBT4T and PDTBT-TT. The crystallization of the two polymers and their polymorphism are controled by changing (i) the rubbing temperature $\left(\mathrm{T}_{\mathrm{R}}\right)$ or (ii) the annealing temperature $\left(\mathrm{T}_{\mathrm{A}}\right)$ of the rubbed films. Indeed, rubbing can be performed up to $\mathrm{T}=230^{\circ} \mathrm{C}$ and thermal annealing is required to reach crystallization temperatures close to the melting. The temperature dependence of the semi-crystalline lamellar morphology is investigated by TEM in Bright Field (BF). A segregated stacking of the comonomers in the chains of PBT4T and PDTBT-TT is identified and the aggregate band at $695-697 \mathrm{~nm}$ is its characteristic 
fingerprint. The thermal stability of this polymorph is investigated by TEM and a correlation with the presence of the aggregate-band at $695 \mathrm{~nm}$ is made. In PBT4T, the loss of segregated stacking correlates perfectly with the loss of the aggregate band whereas in PDTBT-TT the segregated stacking is stable even for temperatures close to the melting. Finally, electron diffraction is used to propose structural models of the two polymers that confirm the segregated stacking.

\section{Experimental section.}

\section{a) Materials}

All reagents and chemicals were purchased from Aldrich and TCI. Toluene (ACS grade) was distilled from $\mathrm{Na}$ /benzophenone. The synthesis of the 2-(trimethylstannyl)-4-(2octyldodecyl)thiophene, 2-(trimethylstannyl)-4-(dodecyl)thiophene, 2-(trimethylstannyl)4-(2-ethylhexyl)thiophene and 2-5-bis-trimethylstannyl-thieno[3,2-b]thiophene blocks follows the procedure described in the literature. ${ }^{5,29}$ The detailed synthesis of PDTBT-TT is further described in the supporting information (see Figure S1). The polymer PDTBT-TT was characterized by Size Exclusion Chromatography, yielding $M_{n}=65 \mathrm{~kg} / \mathrm{mol}, M_{w}=130$ $\mathrm{kg} / \mathrm{mol}$ and PDI $=2.0$. PBT4T was purchased from One Material. It is characterized by $\mathrm{M}_{\mathrm{w}}$ $=80-150 \mathrm{~kg} / \mathrm{mol}$ and a polydispersity index of 2.0-2.5 (measured against PS standard).

\section{b) Oriented thin film preparation by high temperature rubbing.}

High temperature rubbing is performed following the procedure described in the literature. ${ }^{22-24}$ Thin polymer films (20-50 nm thickness) are doctor-bladed from a 5 $\mathrm{mg} / \mathrm{ml}$ ortho-dichlorobenzene solution on clean glass substrates. For the orientation of the films, a home-made rubbing machine is used in a glove box (Jacomex) under 
nitrogen atmosphere. It is composed of a rotating cylinder ( $4 \mathrm{~cm}$ diameter) covered by a microfiber cloth. Rubbing is performed by applying the rotating cylinder with a 2 bar pressure on the translating sample holder $(1 \mathrm{~cm} / \mathrm{s})$. The sample holder can be heated at a controlled temperature (rubbing temperature $\mathrm{T}_{\mathrm{R}}$ ). The sample temperature is allowed to equilibrate for 1-2 min before rubbing. In the following, $\mathrm{R}$ designates the rubbing direction. Post-alignment thermal annealing was done for 10 min in a THMS600 Linkam hot stage under nitrogen atmosphere at the required annealing temperature $\mathrm{T}_{\mathrm{A}}$. Heating and cooling rates were $30{ }^{\circ} \mathrm{C} / \mathrm{min}$ and $20{ }^{\circ} \mathrm{C} / \mathrm{min}$, respectively. Polarized UV-vis absorption spectroscopy was performed using a Varian Carry 5000 UV-VIS-NIR spectrometer with polarized incident light.

d) Transmission Electron Microscopy. Oriented areas are identified for TEM analysis by polarized optical microscopy (POM) using a Leica DMR-X microscope. The polymer films are coated with a thin amorphous carbon film and removed from the glass substrate by floating on a diluted aqueous HF solution (5 wt\%) and subsequently transferred to TEM copper grids. TEM is performed in bright field, high resolution and diffraction modes using a CM12 Philips microscope equipped with a MVIII (Soft Imaging System) Charge Coupled Device camera. Calibration of the reticular distances in the ED patterns is made with an oriented PTFE film. Low dose HR-TEM is performed following the conditions given in Reference 30.

e) Molecular modelling is performed on a Silicon Graphic station using the Cerius2 program (Accelrys). A trial-and-error procedure is used to build up simple structural models of PDTBT-TT and PBT4T, following the same methodology as used previously to refine the crystal structures of P3HT, PCPDTBT and PBT7. 31,16,32 For each step of the trial-and-error method, the molecular geometry was optimized using the "clean" 
procedure of the Cerius2 3.0 Program. The published structure of 5,6-difluoro-4,7dithieno-2,1,3-benzothiadiazole was used as a starting point of the 4,7-di-thiophen-2-ylbenzo1,2,5thiadiazole (T-BT-T) monomer structure. ${ }^{33}$ For the single crystal diffraction calculations, a thickness of $10 \mathrm{~nm}$ was used (additional information on this procedure is available in the User's guide on Modeling Environment).

\section{Results and discussion.}

\subsection{Impact of rubbing temperature on film structure and UV-vis absorption.}

\section{a) Film orientation and crystallinity.}

As reported for most PSCs, high temperature rubbing (HTR) yields highly aligned films. Orientation usually improves with the rubbing temperature $T_{R} \cdot 22-27$ In Figure 1, representative POM images under crossed polarizers illustrate the strong birefringence achieved in both PBT4T and PDTBT-TT thin films rubbed at $200-220^{\circ} \mathrm{C}$. This alignment is further manifested in the polarized UV-vis spectra of the films as seen in Figure 1.d and 1.h. For the incident light polarization POL//R, the spectra consist of a marked vibronic structure with two dominant components noted $0-0$ and $0-1$. Their positions are similar for both polymers (698 nm and $640 \mathrm{~nm}$ for PDTBT-TT, 696nm and $636 \mathrm{~nm}$ for PBT4T). Remarkably, the UV-vis spectra for POL $\perp$ R does not show such a vibronic structure but it is strongly blue-shifted to approx. $600 \mathrm{~nm}$. In accordance with earlier reports on semi-crystalline films of P3HT and PBTTT oriented by HTR, the blue shift in the spectra for POL $\perp R$ is due to the fact that the absorption stems mainly from the 
amorphous domains with randomly oriented and coiled-like chain conformation. Evidence for a regular lamellar structure will be given by TEM (see below).

(a)

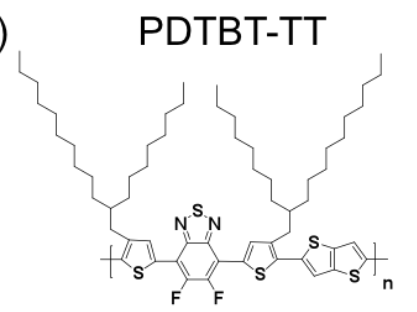

(b) $\mathrm{T}_{\mathrm{R}}=220^{\circ} \mathrm{C}$

(c)

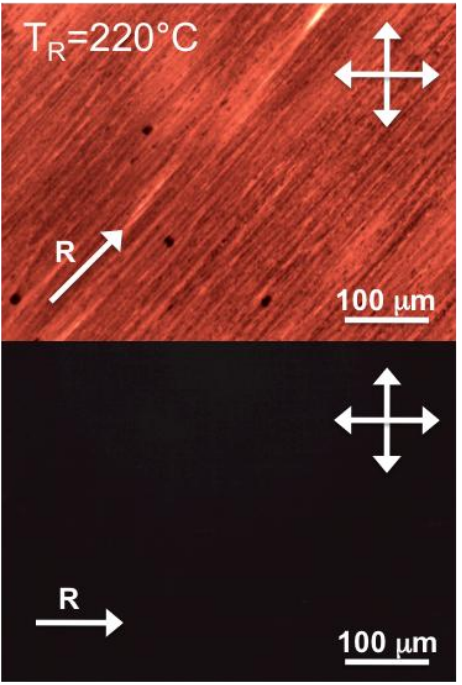

(d)

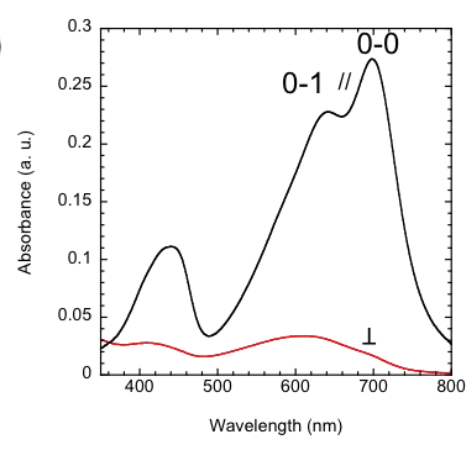

(e)

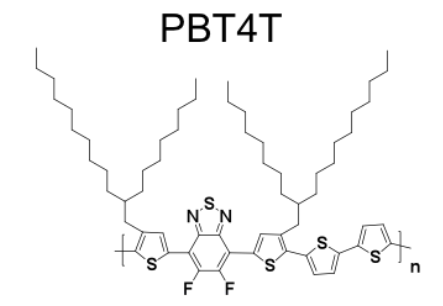

(f)

(g)

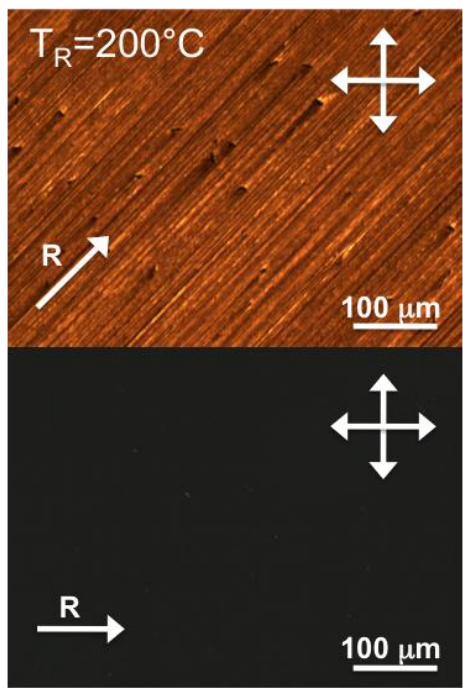

(h)

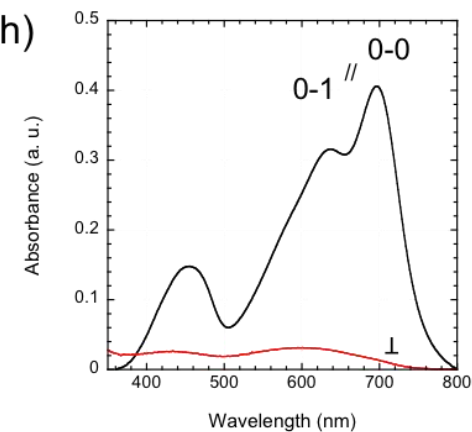

Figure 1. Chemical structures of the two polymers ((a) PDTBT-TT and (e) PBT4T). Polarized Optical Microscopy images (with crossed polarizers) of rubbed thin films showing the high birefringence of the rubbed layers $\left(T_{R}=200-220^{\circ} \mathrm{C}\right)$ for PDTBT-TT $(b$ \& $c)$ and PBT4T ( $f \& g$ ). The arrow indicates the direction of rubbing R. Polarized absorption spectra of the aligned films of PDTBT-TT (d) and PBT4T (h) recorded for the incident light polarization oriented parallel (//, black curves) and perpendicular ( $\perp$, red curves) to the rubbing direction $R$. 
Very high dichroic ratii DR are measured for optimal rubbing temperatures. DR = 17 is obtained for PDTBT-TT rubbed at $220^{\circ} \mathrm{C}$ and $\mathrm{DR}=30$ for PBT4T rubbed at $200^{\circ} \mathrm{C}$ which translate to 3D order parameters $S$ (S defined as $S=(D R-1) /(D R+2))$ of 0.84 and 0.91, respectively. Such large values of S indicate orientations that exceed by far the alignment of films oriented by stretch-alignment ${ }^{34}$ or blade coating. ${ }^{35-37}$ In the present case, the alignment improves substantially when $T_{R}$ increases and reaches a plateau above $210^{\circ} \mathrm{C}$ (see Figure S2).

The anisotropy in optical absorption is directly related to the structure of the aligned films. As for P3HT, the unit cell axes are defined such that $\mathbf{a}$ is along the alkyl side chains, $\mathbf{b}$ is along the $\pi$-stacking and $\mathbf{c}$ is the chain direction. Figure 2 shows the evolution of the electron diffraction (ED) pattern with the rubbing temperature $T_{R}$ for both PBT4T and PDTBT-TT. Initially, the ED pattern of doctor-bladed films consist of a single Scherrer ring of the 020 reflection $\left(\mathrm{d}_{020}=3.6 \pm 0.1 \AA\right.$ for PDTBT-TT and $\mathrm{d}_{020}=3.8 \pm 0.1 \AA$ for PBT4T). The films consist of edge-on crystals with random in-plane orientation. For PBT4T, the onset of orientation is only observed for $\mathrm{T}_{\mathrm{R}}=200^{\circ} \mathrm{C}$. It is manifested by the appearance of equatorial h 00 reflections $(h=1-4)$ whereas the initial 020 ring disappears. Rubbing transforms the randomly oriented edge-on crystals to oriented face-on crystals, as observed previously for P3HT and (poly(2,5-bis(3-dodecyl2-yl)thieno[3,2-b]thiophene)) (PBTTT). ${ }^{13,14}$ For PDTBT-TT, alignment sets in at lower $\mathrm{T}_{\mathrm{R}}$ $\left(150^{\circ} \mathrm{C}\right)$. As $\mathrm{T}_{\mathrm{R}}$ increases, one equatorial 020 is also observed and indicates that a population of aligned edge-on crystals is formed at high rubbing temperatures $\left(\mathrm{T}_{\mathrm{R}} \geq 200^{\circ} \mathrm{C}\right)$. Similar trends were observed for most semi-condcuting polymers aligned by rubbing e.g. PBTTT, P3HT and PTB7 (polythieno[3,4-b]-thiophene-cobenzodithiophene). ${ }^{22-27}$ 
Beside the equatorial reflections, the ED patterns of both polymers display in the direction of rubbing characteristic $00 \mathrm{l}$ reflections, mainly 004 and 0066 reflections. For PDTBT-TT, $\mathrm{d}_{004}=4.4 \pm 0.1 \AA \AA$ and for PBT4T, $\mathrm{d}_{004}=4.8 \pm 0.1 \AA$. Accordingly, the chain axis repeat distances are $c=17.7 \pm 0.4 \AA$ for PDTBT-TT and $c=19.2 \pm 0.4 \AA$ for PBT4T. In PBT4T, additional 005 and 008 reflections are also observed. As seen in Figure 2, 001 reflections are more intense and sharper for PDTBT-TT than for PBT4T, indicating a higher crystalline order in PDTBT-TT.

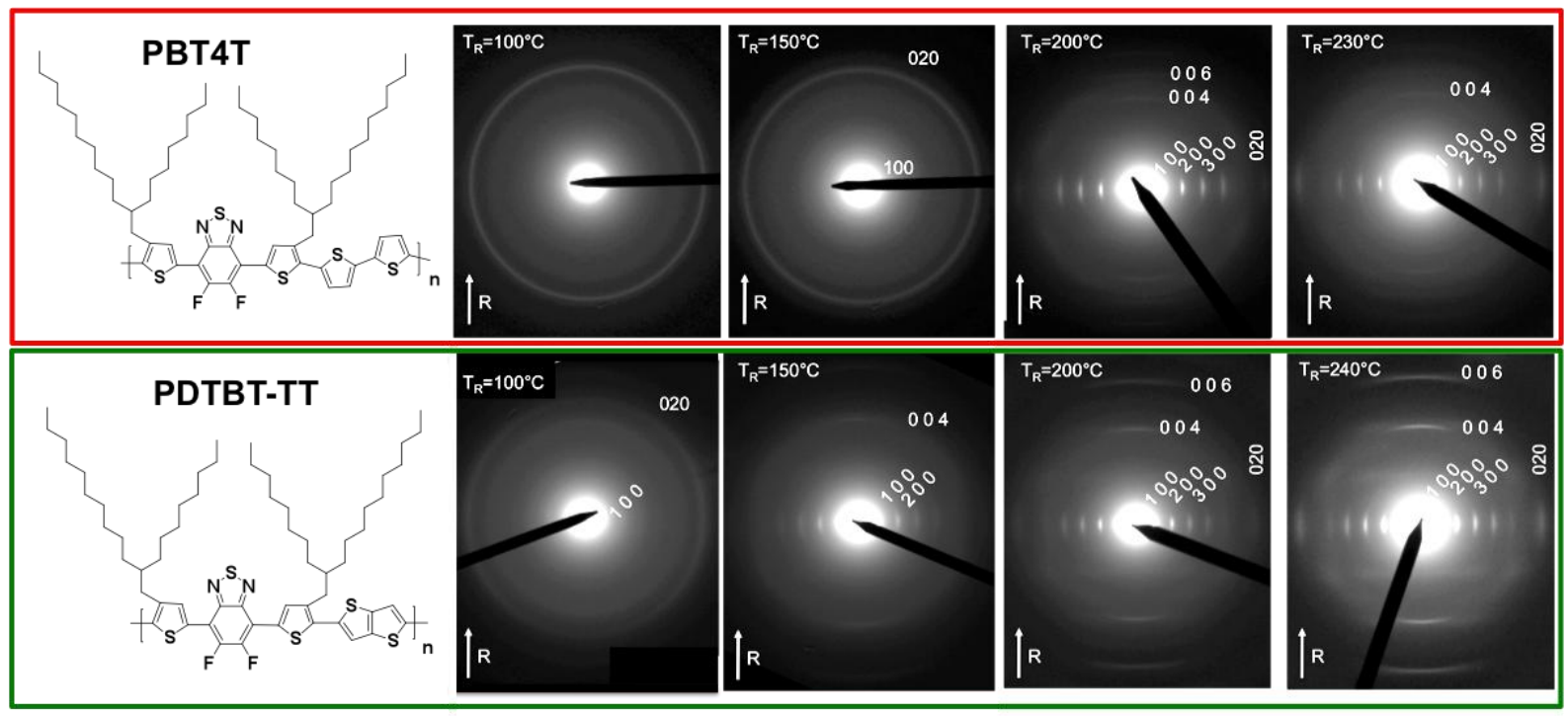

Figure 2. Evolution of the electron diffraction pattern in rubbed thin films of PDTBT-TT and PBT4T as a function of the rubbing temperature $T_{R}$. The arrow points at the rubbing direction $(R)$ of the films as identified by defocused electron diffraction. Please note the difference in the intensity of the 004 and 006 reflections that characterizes the stacking order along the chain direction for PBT4T vs PDTBT-TT.

Analysis of the temperature-dependence of the intensity of the 004 reflection reveals significantly different behaviours of the two polymers in terms of crystalline order along the chain direction. To be more specific, the meridional section profiles as a function of $\mathrm{T}_{\mathrm{R}}$ for both polymers are shown in Figure 3. For PDTBT-TT, the 004 
reflection intensity clearly increases with $T_{R}$ and becomes sharper, indicating higher crystalline order along the chain direction at high $\mathrm{T}_{\mathrm{R}}$, as indicated also by the appearance of diffuse mixed index (hkl) reflections, especially near 302. By contrast, in PBT4T, the 004 reflection intensity decreases drastically beyond $T_{R}=200^{\circ} \mathrm{C}$. Note also that the ED patterns of both polymers lack well defined mixed index reflections. The side chains are therefore disordered contrary to poly(3-(2,5-dioctylphenyl)thiophene) for which the octyl side chains are interdigitated in a perfect crystalline sublattice. ${ }^{38}$
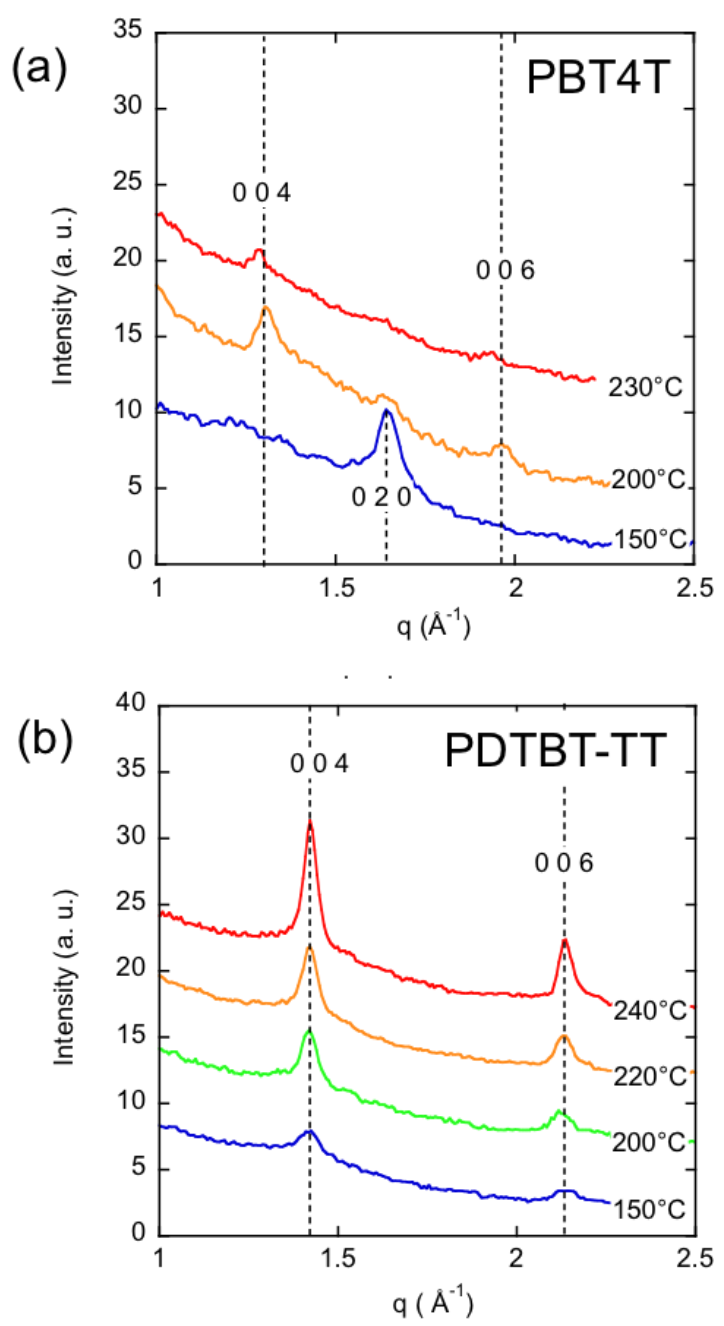

Figure 3. Evolution of section profile along the meridian of the electron diffraction patterns in PBT4T (a) and PDTBT-TT (b) as a function of the rubbing temperature $\left(T_{R}\right)$. Note the different variation of the 004 intensity versus $T_{R}$. 


\section{b) Impact of rubbing temperature $T_{R}$ on optical properties.}

The structural evolution with increasing $\mathrm{T}_{\mathrm{R}}$ results in important changes in the UV-vis absorption spectrum of the rubbed films (see Figure 4). Increasing $\mathrm{T}_{\mathrm{R}}$ impacts strongly the vibronic structure of the absorption spectrum for POL//R as illustrated by the evolution of the ratio $A_{0-0} / A_{0-1}$ with $T_{R}$. For PDTBT-TT, $A_{0-0} / A_{0-1}<1$ for $T_{R}=150^{\circ} \mathrm{C}$ for films that are not well oriented but increases steadily to a value of 1.2 for $\mathrm{T}_{\mathrm{R}}=230^{\circ} \mathrm{C}$. For PTB4T, the situation is different: $A_{0-0} / A_{0-1}$ is aleardy larger than 1 for $T_{R}=150^{\circ} \mathrm{C}$ and reaches a maximum at 1.3 for $\mathrm{T}_{\mathrm{R}}=200^{\circ} \mathrm{C}$ before decreasing again.

The parallel between the evolution of crystalline order and the 0-0 component associated to the aggregate state as a function of $\mathrm{T}_{\mathrm{R}}$ is instructive. For PDTBT-TT, the crystalline order along the chain backbone improves with $\mathrm{T}_{\mathrm{R}}$ up to $230^{\circ} \mathrm{C}$ and at the same time the 0-0 component increases steadily. Usually, in polymers such as P3HT or MEH-PPV, the amplitude amplification of the 0-0 component in absorption relates to an increase in conjugation length or, said differently, an extension of the planarized chain segments. ${ }^{10,27}$ PBT4T illustrates the same pattern, but the trend with $\mathrm{T}_{\mathrm{R}}$ is opposite. The crystalline order along the chain direction decreases as $\mathrm{T}_{\mathrm{R}} \geq 230^{\circ} \mathrm{C}$ and the intensity of the 0-0 component decreases. A similar correlation between the intensity of the 0-0 and crystalline order along the chain direction has been made recently for P3HT. ${ }^{27}$ As seen hereafter, this correlation is further validated by the variation of crystal structure and UV-vis absorption when annealing the thin films. 

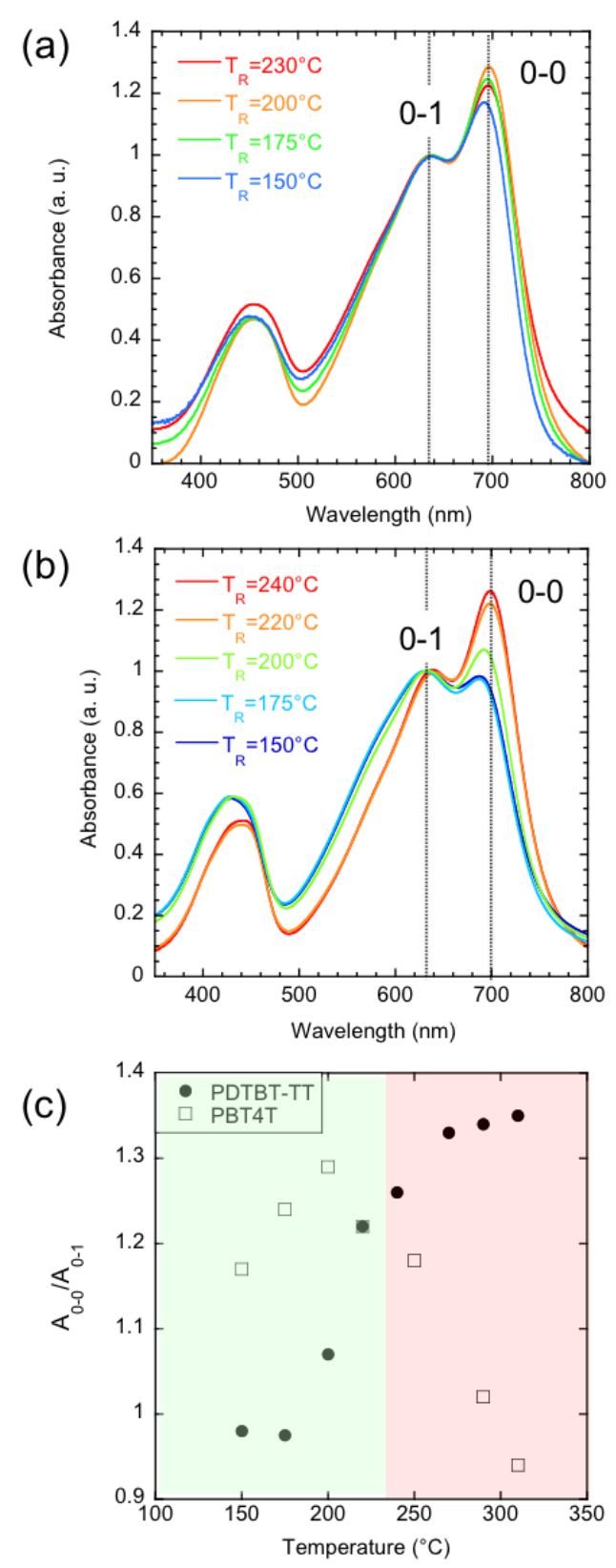

Figure 4. Evolution of the UV-vis absorption spectra in rubbed thin films of (a) PBT4T and (b) PDTBT-TT as a function of the rubbing temperature $T_{R}$. The spectra have all been normalized to the intensity of the 0-1 component of the vibronic structure. (c) Evolution of the ratio between the amplitude of the 0-0 and 0-1 components of the vibronic structure in the UV-vis spectra of PTB4T and PDTBT-TT. The points obtained for $T \leq 220^{\circ} \mathrm{C}$ (light green panel) correspond to films rubbed at various $T_{R}$ whereas for $T \geq 250^{\circ} \mathrm{C}$ (light pink panel), they correspond to the films rubbed at $200-220^{\circ} \mathrm{C}$ and subsequently annealed at $T_{A}$. 


\subsection{Impact of thermal annealing on the structure of the thin films.}

\section{a) Lamellar morphology.}

As-rubbed pristine films of PDTBT-TT and PTB4T have no periodic lamellar structure. This is in strong contrast with P3HT, for which a lamellar morphology is observed for $\mathrm{T}_{\mathrm{R}} \geq 144^{\circ} \mathrm{C}$ with a lamellar periodicity increasing with rubbing temperature. ${ }^{27}$ This lamellar structure becomes only apparent after thermal annealing at $250^{\circ} \mathrm{C} \leq \mathrm{T}_{\mathrm{A}} \leq 290^{\circ} \mathrm{C}$ in PDTBT-TT and PTB4T. As seen in Figure 5, thin films of PDTBTTT display a lamellar morphology with a periodic contrast modulation between the diffracting crystalline domains and the non diffracting interlamellar amorphous zones. For films annealed at $250^{\circ} \mathrm{C}$, the lamellar periodicity (crystalline plus amorphous) is $\mathrm{l}=25 \pm 2 \mathrm{~nm}$. The HR-TEM image in Figure $5 . \mathrm{b}$ shows fringed patterns that indicate faceon crystals. The fringe periodicity of $2.3 \pm 0.1 \mathrm{~nm}$ is consistent with the $\mathrm{d}_{100}$ spacing in the ED pattern. It corresponds to the spacing between $\pi$-stacked conjugated backbones separated by layers of alkyl side chains. The statistical length of chain segments in the crystals is measured at $10 \pm 3 \mathrm{~nm}$ for $\mathrm{T}_{\mathrm{A}}=250^{\circ} \mathrm{C}$ which implies an average width of amorphous interlamellar zones of approx. $15 \mathrm{~nm}$. Accordingly, both bright field and HRTEM illustrate clearly a semi-crystalline morphology of PDTBT-TT reminiscent of the P3HT films. ${ }^{13,14}$ As for P3HT, the lamellar periods 1 increase substantially with the rubbing temperature $\mathrm{T}_{\mathrm{R}}$, from $25 \pm 2 \mathrm{~nm}$ at $250^{\circ} \mathrm{C}$ to $50 \pm 5 \mathrm{~nm}$ at $290^{\circ} \mathrm{C}$. For most semicrystalline polymers, $\mathrm{l}^{-1} \propto\left(\mathrm{T}_{\mathrm{m}}{ }^{0}-\mathrm{T}_{\mathrm{C}}\right)$ where $\mathrm{T}_{\mathrm{m}}{ }^{0}$ is the equilibrium melting temperature and $\mathrm{T}_{\mathrm{C}}$ is the crystallization temperature. ${ }^{39}$ In the present case, the temperature of crystallization corresponds to the annealing temperature and can be used in a Hoffmann-Weeks typee of analysis. ${ }^{40}$ As seen in Figure 5.e, ploting $1 / 1$ versus $\left(\mathrm{T}_{\mathrm{m}}{ }^{0}-\mathrm{T}_{\mathrm{A}}\right)$ shows the expected linear dependence and yields $\mathrm{T}_{\mathrm{m}}^{0}=333 \pm 17^{\circ} \mathrm{C}$. As usual, this 
extrapolated value of $\mathrm{T}_{\mathrm{m}}{ }^{0}$ is higher than the experimental melting temperature $\mathrm{T}_{\mathrm{m}}$ measured by DSC $\left(273.6^{\circ} \mathrm{C}\right.$ for PDTBT-TT). ${ }^{28}$ Also, the increase in lamellar period with $\mathrm{T}_{\mathrm{R}}$ for PDTBT-TT is consistent with the sharpening of the 004 reflection discussed earlier. This result further demonstrates that polymer semiconductors do behave similarly to classical polyolefins regarding the dependence of the melting equilibrium temperature $\mathrm{T}_{\mathrm{m}}^{0}$ with undercooling.
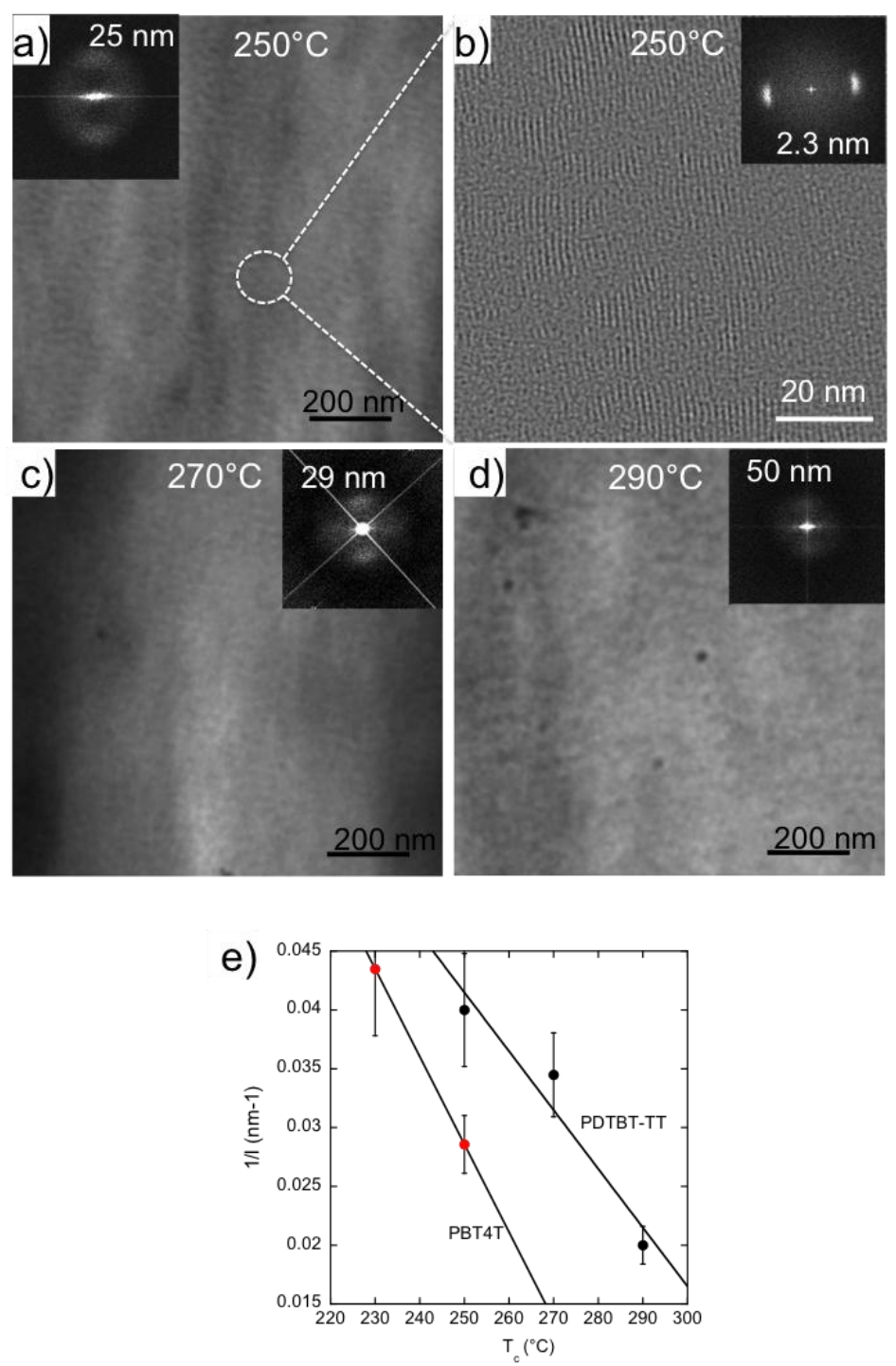

Figure 5. Bright Field TEM images showing the semi-crystalline lamellar structures in aligned thin films of PDTBT-TT $\left(T_{R}=200^{\circ} \mathrm{C}\right)$ after annealing at $T_{A}$ for $10 \mathrm{~min}$. (a) $T_{A}=250^{\circ} \mathrm{C}$, 
(c) $T_{A}=270^{\circ} \mathrm{C}$ and (d) $T_{A}=290^{\circ} \mathrm{C}$. (b) is a HR-TEM image of the film annealed at $T_{A}=250^{\circ}$. The inset corresponds to the FFT. The inset in the upper right corner of each BF shows the Fast Fourier Transform used to determine the lamellar periodicity. Similarly, the inset in the HR-TEM image helps determine the $d_{100}$ periodicity. (e) Dependence of the inverse of the lamellar period l as a function of the crystallization temperature (corresponding to the annealing temperature).

For PBT4T, a similar lamellar morphology was observed for temperatures in the $200^{\circ} \mathrm{C}-250^{\circ} \mathrm{C}$ range. For rubbing temperatures $\mathrm{T}_{\mathrm{R}}<200^{\circ} \mathrm{C}$ and for annealing temperatures $\mathrm{T}_{\mathrm{A}} \geq 270^{\circ} \mathrm{C}$, no lamellar morphology was observed. The absence of clear semi-crystalline morphology for $\mathrm{T}_{\mathrm{A}} \geq 270^{\circ} \mathrm{C}$ is consistent with the reduced crystalline order indicated by the disappearance of the 004 and 006 reflections in the ED patterns. As for PDTBT-TT, the lamellar periodicity increases with temperature in PBT4T. Only a rough extrapolation is possible that yields $\mathrm{T}_{\mathrm{m}}{ }^{0}=288^{\circ} \mathrm{C}\left(\mathrm{T}_{\mathrm{m}}=197^{\circ} \mathrm{C}\right)$.

\section{b) Structure and orientation of crystalline lamellae.}

The impact of thermal annealing on the structure of rubbed thin films of PBT4T is very different from that of PDTBT-TT. Figure 6 compares the evolution of the electron diffraction pattern for films of PBT4T and PDTBT-TT annealed at temperatures in the range $250^{\circ} \mathrm{C}-310^{\circ} \mathrm{C}$ and Figure 7 shows the corresponding section profiles along the meridian and the equator to highlight the structural changes in the films. 


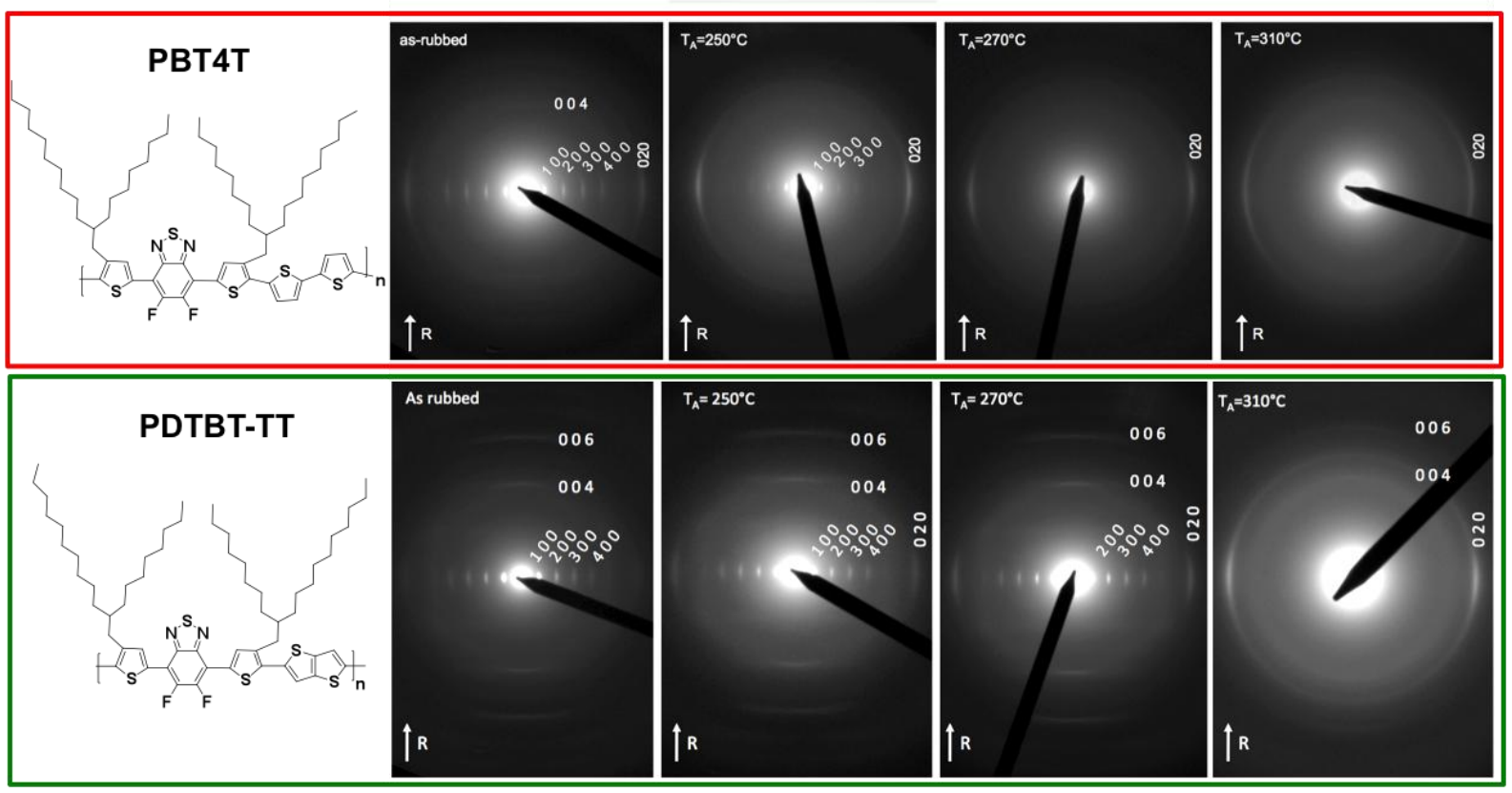

Figure 6. Evolution of the electron diffration patterns of rubbed thin films $\left(T_{R}=200^{\circ} \mathrm{C}\right)$ of PBT4T and PDTBT-TT as a function of the isothermal annealing at different temperatures $T_{A}$. The white arrow indicates the direction of rubbing (R). Note the loss of intensity of the 0 04 and 006 reflections at $250^{\circ} \mathrm{C}$ for PBT4T contrary to PDTBT-TT. 
(a)

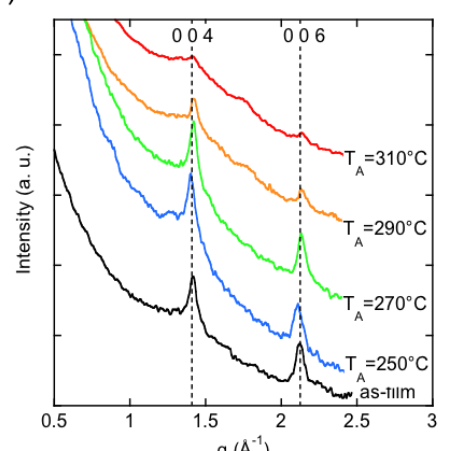

(b)

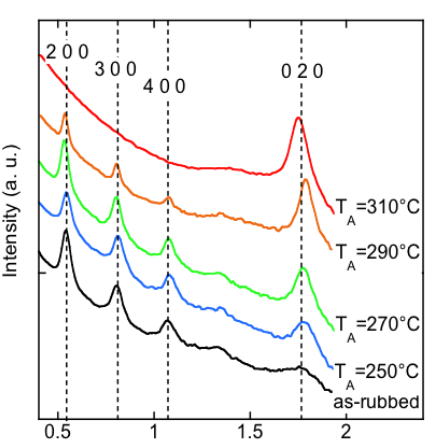

$q\left(\AA^{-1}\right)$

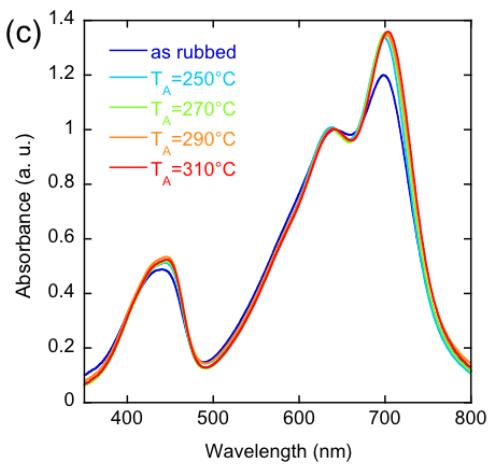

(d)

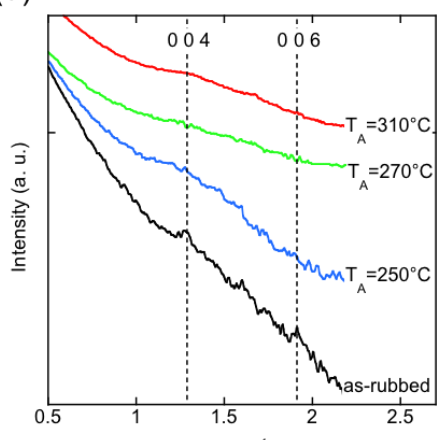

$q\left(\AA^{-1}\right)$

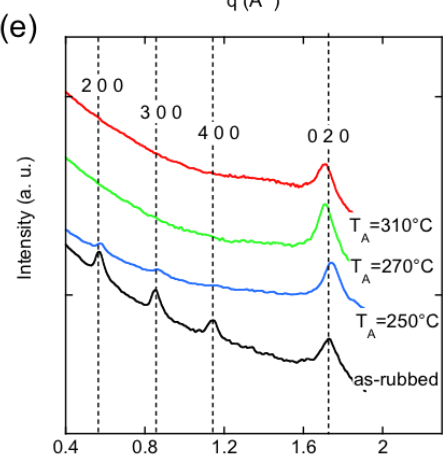

$\mathrm{q}\left(\AA^{-1}\right)$

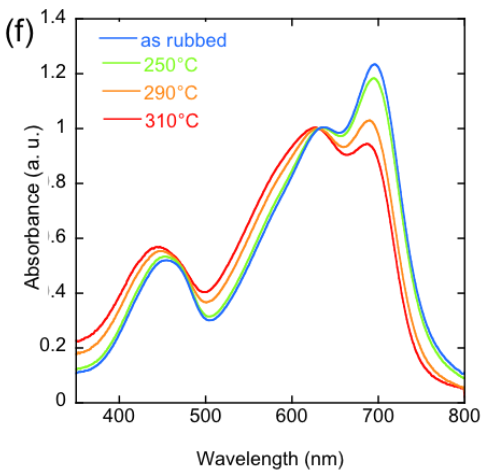

Figure 7. Impact of annealing temperature $T_{A}$ on the crystalline structure in highly oriented films of PDTBT-TT and PBT4T aligned by rubbing at $200^{\circ} \mathrm{C}$. (a) and (d) correspond to the section profile of the ED patterns along the meridian and probe the crystalline order along the chain direction equator. (b) and (e) correspond to the section profile of the ED patterns along the equator and probe crystalline order along the alkyl side chain and/or the $\pi$-stacking directions. (c) and (f) $U V$-vis absorption spectra recorded for the polarization parallel to the rubbing direction. The spectra have been normalized in intensity for the 0-1 component to highlight the evolution in the intensity of the 0-0 component as a function of annealing temperature. 
For PBT4T, annealing the films at $\mathrm{T}_{\mathrm{A}} \geq 250^{\circ} \mathrm{C}$ results in a strong increase in the intensity of the 020 equatorial peak at the expense of the h00 reflections that fully disappear for annealing temperatures $\mathrm{T}_{\mathrm{A}} \geq 270^{\circ} \mathrm{C}$ (see section profile along the equator in Figure 7.b and 7.e). Also, along the meridian, the faint 004 reflection originally seen in the film rubbed at $200^{\circ} \mathrm{C}$ is lost, even after annealing at temperatures as low as $250^{\circ} \mathrm{C}$ (see Figure 7.d). Accordingly, in PBT4T, annealing at $\mathrm{T}_{\mathrm{A}} \geq 250^{\circ} \mathrm{C}$ causes a reorientation of aligned face-on crystals to aligned edge-on crystals and tends to suppress crystalline perfection along the chain direction as indicated by the loss of all 001 reflections.

The thermal annealing has a different impact on the structure of PDTBT-TT films. First, the crystalline order in the films probed along the chain direction (section profile 7.a) is maintained up to very high annealing temperatures $\mathrm{T}_{\mathrm{A}}$ close to the melting temperature. Indeed, the 004 and 006 reflections are still present in films annealed at $\mathrm{T}_{\mathrm{A}}$ in the range $270^{\circ} \mathrm{C}-310^{\circ} \mathrm{C}$. Alignment is not fully lost for $\mathrm{T}_{\mathrm{A}}=310^{\circ} \mathrm{C}$ but the in-plane orientation distribution is broadened. Second, the preferential face-on contact plane of PDTBT-TT crystals remains up to $\mathrm{T}_{\mathrm{A}}=270^{\circ} \mathrm{C}$ as indicated by the presence of well defined h 00 reflections. These reflections coexist with the equatorial 020 reflection arising from aligned crystals that changed their contact plane from face-on in as-rubbed films to edge-on after annealing. For $\mathrm{T}_{\mathrm{A}}>270^{\circ} \mathrm{C}$, the intensity of the 020 equatorial peak becomes dominant, indicating that the films of PDTBT-TT consist of a majority of aligned edge-on crystals. For $\mathrm{T}_{\mathrm{A}}=310^{\circ} \mathrm{C}$, no more face-on crystals of PDTBT-TT are evidenced, the films consist exclusively of aligned edge-on crystals. 


\section{c) Evolution of UV-Vis spectra with annealing temperature.}

As is the case for the rubbing temperatures, the annealing temperature has a major impact on the UV-vis absorption spectrum of the thin films. Figure 7.c and 7.f show the evolution of the UV-vis absorption (for POL//R) as a function of annealing temperature for PDTBT-TT and PBT4T, respectively.

For PDTBT-TT, the intensity of the 0-0 component increases with the annealing temperature up to the melting temperature (see Figure 7.c). Moreover, a shoulder that corresponds most likely to the $0-2$ becomes visible in the spectrum for $\mathrm{T}_{\mathrm{A}}=310^{\circ} \mathrm{C}$. There is also a small red-shift of the high energy absorption band from 442.5 to $447.5 \mathrm{~nm}$. This red shift is apparently related to the overlap of two contributions with an increase in intensity of the low energy component at $447.5 \mathrm{~nm}$ as $\mathrm{T}_{\mathrm{A}}$ increases. The plot of the ratio $A_{0-0} / A_{0-1}$ versus temperature $\left(T_{R}\right.$ and $\left.T_{A}\right)$ in Figure 4.c shows a steady increase of the ratio $A_{0-0} / A_{0-1}$ with increasing temperature $\left(T_{R}\right.$ or $\left.T_{A}\right)$ in PDTBT-TT until some saturation is observed at higher temperatures. This evolution is usually related to increased planarization, which is also consistent with enhanced stacking order observed along the chain direction by ED.

The situation is different in PBT4T. In these films, increasing $\mathrm{T}_{\mathrm{A}}$ from $250^{\circ} \mathrm{C}$ to $310^{\circ} \mathrm{C}$ results in a clear decrease of intensity of the $0-0$ component. In addition, the $0-1$ component is blue-shifted and a shoulder at $\sim 590 \mathrm{~nm}$ appears at higher $\mathrm{T}_{\mathrm{A}}$. The high energy component shows a small blue-shift with increasing $\mathrm{T}_{\mathrm{A}}$ from $455 \mathrm{~nm}$ to $445 \mathrm{~nm}$. As seen in Figure 4.c, thermal annealing results in a drastic decrease of the ratio $\mathrm{A}_{0-0} / \mathrm{A}_{0-1}$ with increasing $\mathrm{T}_{\mathrm{A}}$. Accordingly, both PDTBT-TT and PBT4T show opposite trends in the intensities of the aggregate band at $697 \mathrm{~nm}$ and also opposite trends in terms of band shifting. 
As was the case when evaluating the impact of $\mathrm{T}_{\mathrm{R}}$ on the UV-vis absorption of PBT4T and PDTBT-TT, making the parallel between the structural and the UV-vis spectrum evolutions versus $\mathrm{T}_{\mathrm{A}}$ is again highly instructive. In fact, the trends observed for both polymers with $\mathrm{T}_{\mathrm{A}}$ confirm the previous observations versus $\mathrm{T}_{\mathrm{R}}$. Indeed, in PDTBT-TT, the order along the chain axis is correlated with an increase of the intensity of the $0-0$ vibronic component in the UV-vis spectrum. For PBT4T, the situation is opposite. When crystalline order along the chain direction is destroyed for $\mathrm{T}_{\mathrm{A}} \geq 250^{\circ} \mathrm{C}$, the $0-0$ component decreases in intensity. It is worth to stress that the presence of the 0-0 vibronic component does not correlate with the order observed along the $\pi$-stacking direction. Indeed, for both polymers, the intensity of the 020 associated with the $\pi$-stacking is observed for high values of $\mathrm{T}_{\mathrm{A}}$ and $\mathrm{T}_{\mathrm{R}}$. Accordingly, the intensity of the 0-0 component correlates with the extension of planarized chain segments in the crystalline domains of the two polymers. However, at this point, the type of stacking mode in both polymers (segregated versus mixed) remains to be uncovered. To that aim, the next section presents the result of the structure modeling of the two polymers based on electron diffraction.

\subsection{Modeling of the structures.}

The structure of PDTBT-TT was modeled following the same approach as proposed in our previous studies on PCPDTBT and F-PCPDTBT. ${ }^{15}$ In PCPDTBT, the cyclopentadithiophene and the benzothiadiazole units stack in a segregated mode, suggesting that a similar situation could exist in PDTBT-TT and PBT4T.

The structure of PDTBT-TT was modeled by using an orthorhombic unit cell (see Figure 8) with $a=24 \AA, b=7.6 \AA$ and $c=17.7 \AA$ and P2 symmetry (P21 symmetry did not yield to a good agreement of calculated vs experimental ED patterns). The conformation 
of the chain was such that the two thiophenes on either side of the TT (thienothiophene) or the BT (benzothiadiazole) are in trans-cis conformation (see Figure 9). This conformation of T-BT-T block prevails in the structure of 5,6-difluoro-4,7-dithieno2,1,3-benzothiadiazole (approx. 63\%). ${ }^{33}$ The resulting chain conformation for PDTBT-TT is different from the zig zag conformation calculated by Zhang et al. using DFT. ${ }^{28}$ In their model, the T-BT-T block is in cis,cis conformation whereas in the oligomer crystal of Nielsen et al. the trans,cis conformation is predominant. ${ }^{33}$ Choosing the cis,cis conformation of the T-BT-T unit results in a zig zag chain conformation with a $\mathbf{c}$ axis parameter that is twice the experimentally observed one. Indeed, the strong reflections on the meridian are indexed as 004 and 006 with c=17.7 $\AA$ and there is no evidence for a doubling of the c axis period. In addition, it must be stressed that the orientation of the BT in the chain is usually subjected to statistical disorder as observed previously in PCPDTBT and also in oligomers containing BT. ${ }^{41}$ In the modeling of the structure, the unit cell contains two chains so that the relative shift along the $\mathrm{c}$ axis (chain direction) between the chains is used to modulate the intensity distribution in the $\mathrm{l}^{\text {th }}$ layer lines of the ED pattern. No side chains are included in the modeling as the ED patterns do not show well defined mixed index reflections that would indicate a well ordered stacking of side chains. ${ }^{38}$ As seen in Figure 8.a, the intensity distribution along the $c^{*}$ axis is well reproduced for a purely segregated stacking of the BT and the co-monomers (Figure 8.c) i.e. dominant h04 and h06 reflections. In the proposed structural model, the a axis parameter is doubled in order to shift every other $(b, c)$ plane by $c / 4$ to effectively interdigitate the alkyl side chains (note that if side chains would be included in the model, they would increase the intensity of the 004 reflection). 


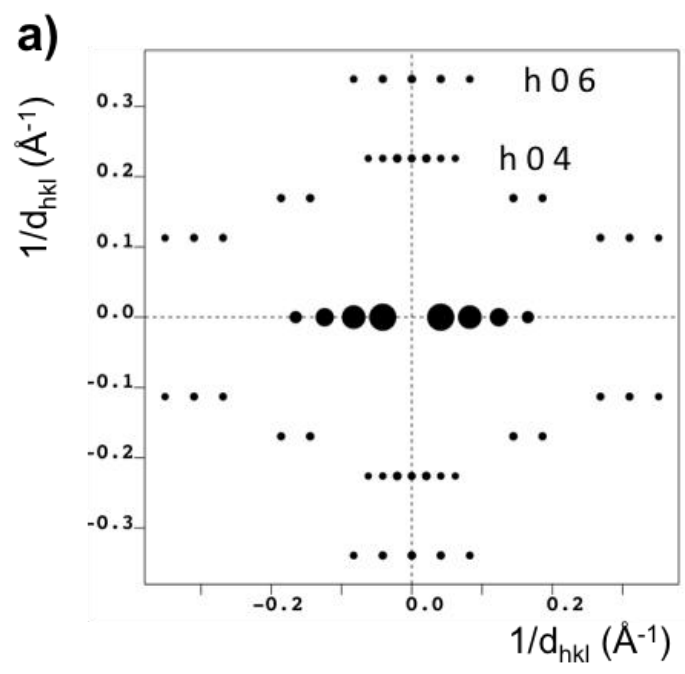

b)
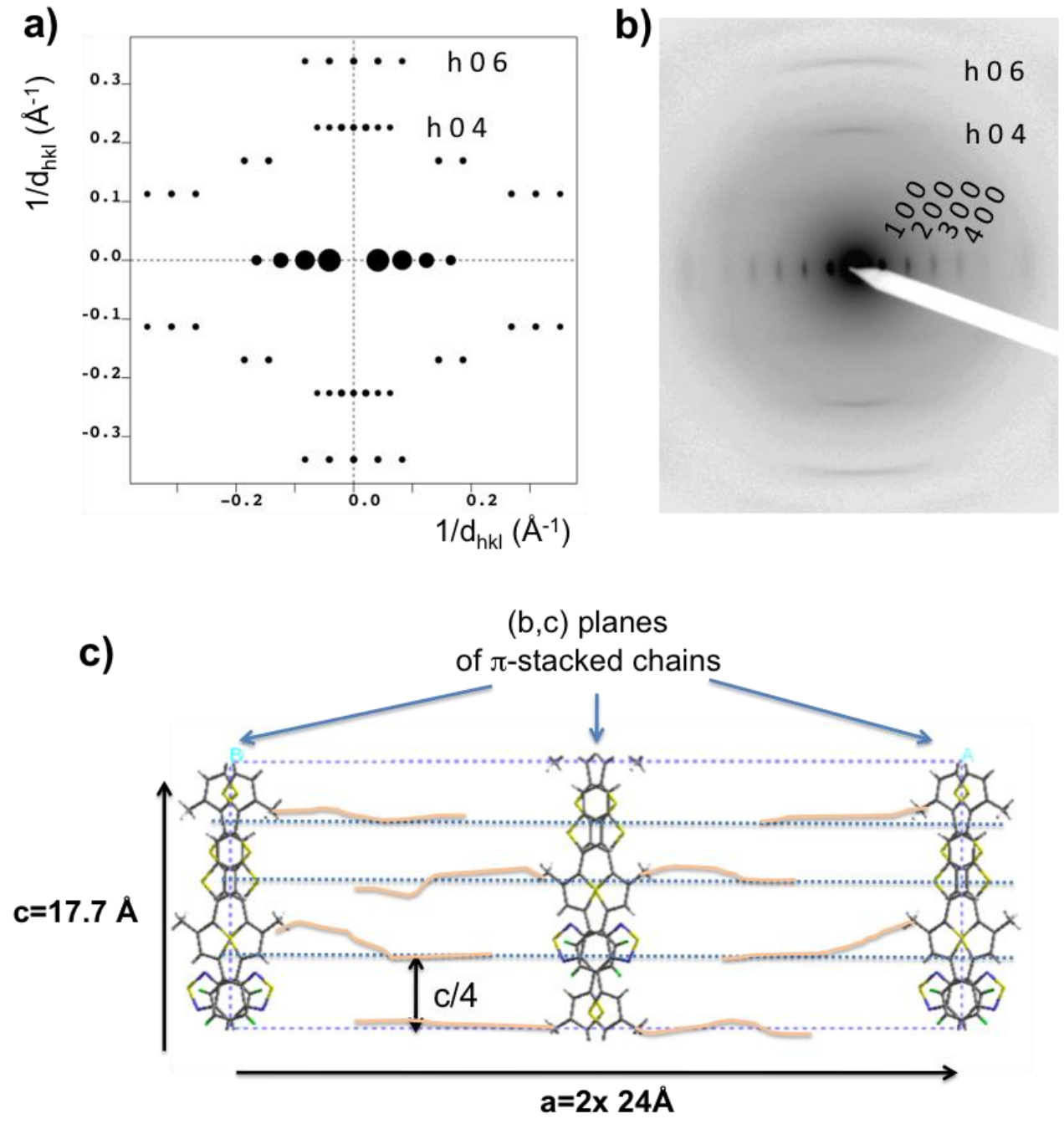

Figure 8. Comparison of the calculated (a) and experimental ED pattern for the [0 10 l zone of PDTBT-TT. (c) (010) projection of the refined model corresponding to a segregated stacking of the chains. The unit cell is doubled along the a axis to take into account the c/4 shift between successive $(b, c)$ planes of $\pi$-stacked chains. In the true structure, the shift between successive $(b, c)$ planes is given by $\pm(2 n+1) c / 4$ ( $n$ is an integer). This disorder is expected to induce a streaking of the 004 and the 006 reflections (see text) in the direction parallel to $a^{*}$ and the disappearance of the (h 03 3) and (h 02 2) reflections as observed in the experimental pattern in (b).

However, in the thin films, this shift is and must be statistical and equal to $\pm(2 n+1) c / 4$. Such a disorder erases the h 03 and h 02 reflections present in the 
calculated ED pattern of the model in Figure 8.c and causes a streaking of the 004 and 0 06 reflections parallel to a* as observed in the experimental ED. ${ }^{42,43}$ In other words, the elementary building blocks of the PDTBT-TT crystals are individual $(b, c)$ planes in which the chains are $\pi$ stacked in segregated mode i.e. with pure $\pi$-stacks of BT and T-TT-T (see Figure 9).

A similar modeling was also performed for PBT4T using an orthorhombic cell with parameters $a=22.2 \pm 0.5 \AA, \quad b=7.5 \pm 0.5 \AA$ and $c=19.6 \pm 0.5 \AA$. Again, the chain conformation proposed by Zhang was not retained since it leads to a shortening of the c axis due to the zig zag conformation. In addition, such a zig zag conformation results in intense h 01 reflections that are not observed in the present case (confer to the study on PTB7 in reference 32 that shows a zig zag conformation with intense 101 reflections as a fingerprint). In the retained chain conformation, the four thiophenes are in all- trans conformation. As a result the T-BT-T conformation is identical to the trans,cis conformation observed for the oligomer. ${ }^{33}$ The calculated ED pattern supports again a segregated stacking for PBT4T although the calculated ED patterns shows less agreement with the experimental one. A statistical c/4 shift between successive $(b, c)$ planes is also required to achieve an efficient side chain interdigitation (see Figure S3). The discrepancies between experimental and calculated ED pattern, such as overestimated intensities of h05 reflections, are due to the fact that the branched side chains were not included in the calculation of the ED. Indeed, including the side chains in the calculations would strongly enhance the intensity of (h 04 ) reflections that are observed in the experimental ED. 


\section{Discussion.}

Following the work by Spano and coworkers, it is known that the optical properties of conjugated polymers in the crystalline or aggregated state can be either Jor H-type depending on the relative orientation of adjacent polymer chains in the crystals and also on the amount of energetic disorder. ${ }^{10,11}$ In the case of PBT4T, TEM investigations indicate that thin films prepared at $\mathrm{T}_{\mathrm{A}} \geq 250^{\circ} \mathrm{C}$ present reduced crystalline perfection along the chain direction and therefore enhanced structural disorder in the layers of $\pi$-stacked chains. This enhanced disorder translates in a remarkable reduction of the 0-0 low energy component of the absorption spectrum. In strong contrast, for PDTBT-TT, crystalline order along the chain direction is maintained at high processing temperatures and the 0-0 component is the dominant one. For $\mathrm{T}_{\mathrm{A}}=310^{\circ} \mathrm{C}$, the spectrum shows a remarkably well defined vibronic structure that is consistent with highly planarized chain segments in the crystals of the segregated packing of PDTBT-TT. 
PBT4T
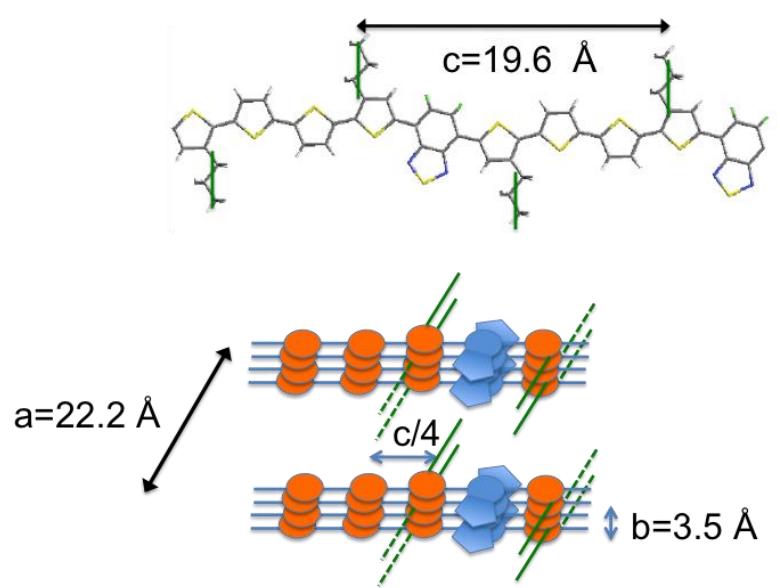

T - T - T - BT - T

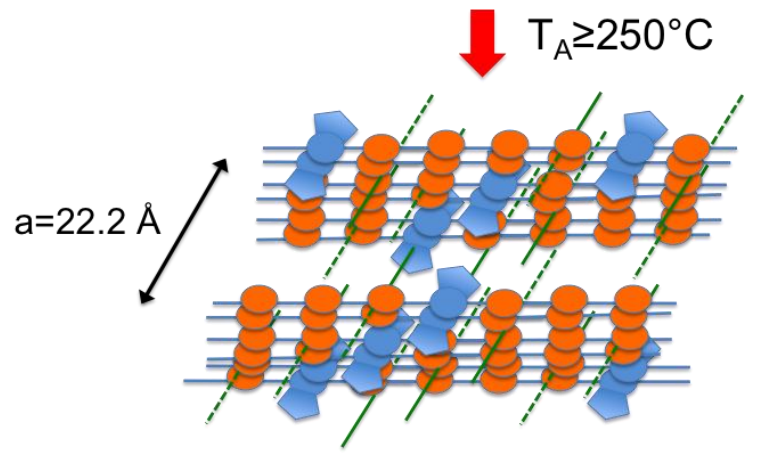

Layered structure with
intra-stack disorder

\section{PDTBT-TT}
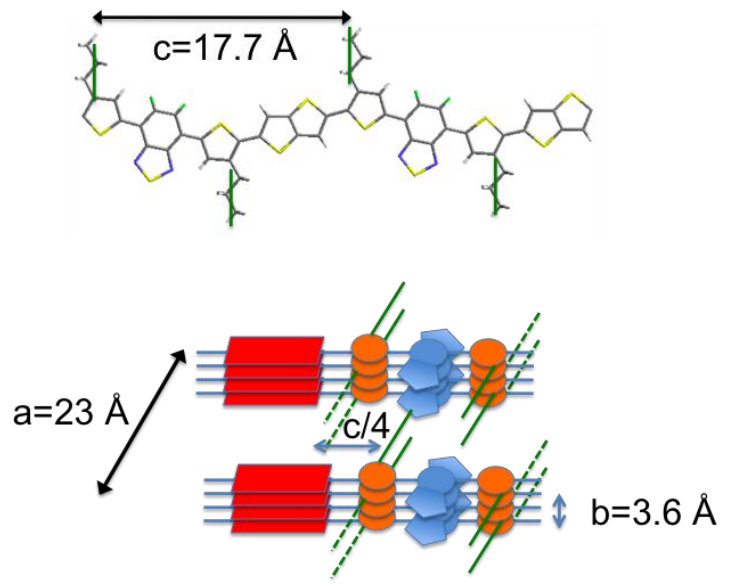

TT - T - BT - T

Stability of segregated

stacks for $T_{A}$ close to the melting temperature

Figure 9. Schematic illustration of the segregated packing in PDTBT-TT and PBT4T. One $(b, c)$ plane over two is statistically shifted by $\pm(2 n+1) c / 4$ to allow side chains to interdigitate. In the trial structure used to model the ED pattern, the unit cell is doubled along the $\boldsymbol{a}$ axis to account for this shift while in the true structure the chift is statistic in essence.

To understand the correlation between optical absorption spectra and crystal packing, the comparison with similar polymers such as p(NDI2ODT2) and PCPDTBT is interesting. The form I of p(NDI2OD-T2) consists of a segregated stacking of the naphthalene bisimide and bithiophene units. ${ }^{6}$ The UV-vis spectra of form I shows also a 
pronounced 0-0 band close to $795 \mathrm{~nm}$. Form II of p(NDI20DT2) involves a mixed stacking of NDI and bithiophene as indicated by a strong $\begin{array}{llllll}0 & 0 & 2\end{array}$ and a weak $\begin{array}{llll}0 & 0 & 1\end{array}$ reflections 6,8 The strength of the $0-0$ band observed in form I is strongly reduced for the films of p(NDI20DT2) in form II. A similar behaviour is observed for PBT4T. The case of PCPDTBT bears also strong analogy and helps in the analysis of PBT4T vs PDTBT-TT. In PCPDTBT, various polymorphs were identified. ${ }^{15}$ One of them shows large scale $\pi$ stacking and a strong aggregate band. ${ }^{44}$ A second polymorph of PCPDTBT is obtained by solvent vapor annealing in chlorobenzene. This thermodynamically stable structure consists of a high symmetry packing of dimers of $\pi$-stacked PCPDTBT chains separated from other dimers by alkyl side chains i.e. a structure without long-range $\pi$-stacking. ${ }^{15}$ Interestingly, this structure shows a blue-shifted absorption spectrum without important low energy "aggregate" band. These few examples support the present finding that the strong 0-0 "aggregate" absorption is related to a long-range $\pi$-stacking of highly planarized PDTBT-TT chains forming segregated stacks of T-BT-T and thienothiophene.

The case of PBT4T suggests that a third case exists, namely a "disordered" type of stacking as illustrated in Figure 9. For this polymer, ED shows that for $\mathrm{T} \geq 250^{\circ} \mathrm{C}$, all $00 \mathrm{l}$ reflections disappear. Clearly, there is no well defined registry in the $\pi$-stacked polymer chains along the chain axis in PBT4T for samples prepared at $T \geq 250^{\circ} \mathrm{C}$. Chains are still $\pi$ stacked in layers but there is no more long-range segregation in the stacking of BT and quaterthiophene, as illustrated in Figure 9. As in PCPDTBT, in the high-T phase of PBT4T $\left(\mathrm{T} \geq 250^{\circ} \mathrm{C}\right)$, the BT units may still be associated in pairs (due to dipole-dipole interactions between BTs), but such pairs are stacked in a disordered manner i.e. without positional order along the chain direction. In other words, while PDTBT-TT shows strong positional order in the $(b, c)$ planes of $\pi$-stacked chains, the high-T phase of 
PBT4T does not. The disordered stacking of chains in PBT4T is in turn responsible for the reduced intensity of the $0-0$ aggregate band.

The case of PDTBT-TT is clearly different. The segregated stacking is maintained up to high temperatures and no transition to mixed or randomized stacking is observed. Due to stronger $\pi$-stacking interactions between TTs, the segregated stacking mode is locked and stable up to temperatures close to the melting. Combining the PBT4T and PDTBT-TT cases, the high temperature stability of the segregated stacking in PDTBT-TT can be rationalized in terms of stronger $\pi-\pi$ interactions between the TT blocks. Instead, in PBT4T the T4 unit is subjected to more conformational disorder due to the possible torsion between successive thiophenes. In other words, in PDTBT-TT the TT block favors planarization of the polymer backbone whereas in PBT4T, the quaterthiophene may experience more conformational disorder at high temperature that will impact both the backbone planarization and their $\pi$-stacking in the crystal. The stabilization of a single stacking mode in a large temperature domain is very important as it suppresses the possibility of polymorphism (as observed in p(NDI20DT2)) or the formation of disordered $\pi$-stacks as evidenced in the PBT4T. Avoiding such polymorphism/disordering may be beneficial for corresponding opto-electronic properties.

\section{Conclusion.}

Two high performance polymer semiconductors, PDTBT-TT and PBT4T have been oriented and crystallized by a combination of high temperature rubbing and thermal annealing in order to establish correlations with optical properties. Both, PDTBT-TT and 
PBT4T rubbed films have a semi-crystalline lamellar morphology with a lamellar period l that scales like $1 / \mathrm{l} \propto\left(\mathrm{T}_{\mathrm{m}}{ }^{0}-\mathrm{T}_{\mathrm{A}}\right)$. Both polymers can crystallize in a segregated stacking mode in oriented thin films prepared by HTR. In the segregated mode, the chain $\pi$-stack so as to form pure stacks of the benzothiadiazole and of the co-monomer. This stacking is preferentially obtained in thin films aligned by high temperature rubbing at $\mathrm{T}=200^{\circ} \mathrm{C}$. In PDTBT-TT, the segregated stacking is stable up to very high temperatures close to the melting. Polarized optical absorption clearly indicates that the so-called aggregate band correlates with the perfection of the segregated stacking of the chains in $(b, c)$ planes of the crystals and it disappears when disorder along the chain direction sets in at high temperatures. Accordingly, this study illustrates the importance of the co-monomers selected in the design of low bangap polymers and the way it impacts the polymorphism in thin films and ultimately the optical absorption properties. Further experiments are in progress to determine the role played by polymorphism and stacking disorder on the charge transport in field effect transitors of PBT4T and PDTBT-TT.

\section{Acknowledgments.}

Bernard Lotz is gratefully acknowledged for fruitful discussions and careful reading of the manuscript. C. Blanck and M. Schmutz are gratefully acknowledged for technical support in TEM. S. Grigorian is acknowledged for providing the sample of PCE11. Support from the IRTG Soft Matter and the European community (Rhin Solar Interreg project C25), the Université de Strasbourg, the Investissements d'avenir (IDEX) and the Fédération de recherche Matériaux et Nanosciences Alsace are gratefully acknowledged. ANR is acknowledged for financial support through ANISOTHERM project (ANR-17CE05-0012-04). 


\section{References}

(1) Zhang, Z.-G. and Wang, J. J. Mater. Chem. 2012, 22, 4187.

(2) Boudreault, P.-L. T. ; Najari, A. and Leclerc, M. Chem. Mater. 2011, 23, 456.

(3) Facchetti, A. Materials Today, 2013, 16, 123.

(4) Kularatne, R. S. ; Magurudeniya, H. D. ; Sista, P. ; Biewer, M. C. and Stefan, M. C. J. Polym. Sci. Polym. Chem. A 2013, 51, 743.

(5) (a) Biniek, L.; Fall, S.; Chochos, C. L.; Anokhin, D. V.; Ivanov, D. A.; Leclerc, N.; Lévêque, P.; Heiser, T. Macromolecules 2010, 43, 9779-978. (b) Li, Z.; Zhang, Y.; Tsang, S.-W.; Du, X.; Zhou, J.; Tao, Y.; Ding, J. The Journal of Physical Chemistry C 2011, 115, 1800218009. (c) Osaka, I.; Saito, M.; Koganezawa, T.; Takimiya, K. Advanced Materials 2014, 26, 331338.

(5) Brinkmann, M. ; Gonthier, E. ; Bogen, S. ; Tremmel, K. ; Ludwigs, S. ; Hufnagel, M. ; Sommer, M. ACS Nano, 2012, 6, 10319.

(6) Donley, C. L. ; Zaumseil, J. ; Andreasen, J. W. ; Nielsen, M. M. ; Sirringhaus, H. ; Friend, R. H. ; Kim, J.-S. J. Am. Chem. Soc. 2005, 127, 12890-12899.

(7) Tremel, K. ; Fischer, F. S. U. ; Kayunkid, N. ; DiPietro, R. ; Kiriy, A. ; Neher, D. ; Ludwigs, S. and Brinkmann, M. Adv. Energy Mater., 2014, 4, 1301659.

(8) Brinkmann, M. ; Hartmann, L. ; Biniek, L. ; Tremel, K. and Kayunkid, N. Macromol. Rapid. Comm. 2014, 35, 9.

(9) Leclerc, N.; Chávez, P.; Ibraikulov, O. A.; Heiser, T. and Lévêque, P. Polymers 2016, 8, 11.

(10) Spano, F. C. and Silva, C. Ann. Rev. Phys. Chem. 2014, 65, 477. 
(11) C. Scharsich, F. S. U. Fischer, K. Wilma, R. Hildner, S. Ludwigs and A. Köhler J. polymer Sci. Part B: Polym. Phys. 2015, 53, 1416.

(12) Gross, Y. M.; Trefz, D.; Tkachov, R.; Untilova, V.; Brinkmann, M.; Schulz, G. L.; Ludwigs, S. Macromolecules 2017, 50, 5353-5366.

(13) Crossland, E. J. W. ; Tremel, K. ; Fischer, F. S. U. ; Rahimi, K. ; Reiter, G. ; Steiner, U. and Ludwigs, S. Adv. Mater. 2012, 24, 839.

(14) Crossland, E. J. W. ; Rahimi, K. ; Reiter, G. ; Steiner, U. and Ludwigs, S. Adv. Funct. Mater. 2011, 21, 518.

(15) Rahimi, K.; Botiz, I.; Stingelin, N.; Kayunkid, N.; Sommer, M.; Koch, F. P. V.; Nguyen, H.; Coulembier, O.; Dubois, P.; Brinkmann, M.; Reiter, G. Angewandte Chemie International Edition 2012, 51, 11131-11135.

(16) (a) Fischer, F. S. U.; Kayunkid, N.; Trefz, D.; Ludwigs, S.; Brinkmann, M. Macromolecules 2015, 48, 3974-3982. (b) Fischer, F. S. U.; Trefz, D.; Back, J.; Kayunkid, N.; Tornow, B.; Albrecht, S.; Yager, K. G.; Singh, G.; Karim, A.; Neher, D.; Brinkmann, M.; Ludwigs, S. Advanced Materials 2015, 27, 1223-1228. DeLongchamp, D. M.; Kline, R. J.; Jung, Y.; Germack, D. S.; Line, E. K. ; Moad, A. JL. J. Richter, L. J. ; Toney, M. F.; Heeney, M. and McCulloch, I. ACS Nano, 2009, 3, 780-787.

(18) Müller, C. ; Aghamohammadi, M. ; Himmelberger, S. ; Sonar, P. ; Garriga, M. ; Salleo, A. and Campoy-Quiles, M. Adv. Funct. Mater. 2013, 23, 2368.

(19) Brinkmann, M. and Wittmann, J.-C. Adv. Mat. 2006, 18, 860.

(20) Brinkmann, M. ;Contal, C. ; Kayunkid, N. ; Djuriç, T. and Resel, R. Macromolecules 2010, $43,7604$. 
(21) Brinkmann, M. ; Charoenthai, N. ; Traiphol, R. ; Piyakulawat, P. ; Wlosnewski, J. and Asawapirom, U. ; Macromolecules, 2009, 42, 8298.

(22) Hartmann, L.; Tremel, K.; Uttiya, S.; Crossland, E.; Ludwigs, S.; Kayunkid, N.; Vergnat, C.; Brinkmann, M. Adv. Funct. Mater. 2011, 21, 4047-4057.

(23) Biniek, L.; Leclerc, N.; Heiser, T. and Brinkmann, M. Macromolecules 2013, 46, 4014.

(24) Biniek, L.; Poujet, S.; Djurado, D.; Gonthier, E.; Tremel, K.; Kayunkid, N.; Zaborova, E.; Crespo-Monteiro, N.; Boyron, O.; Leclerc, N.; Ludwigs, S. and Brinkmann, M. Macromolecules, 2014, 47, 3871.

(25) Tremel, K. ; Fischer, F. S. U. ; Kayunkid, N. ; DiPietro, R. ; Kiriy, A. ; Neher, D. ; Ludwigs, S. and Brinkmann, M. Adv. Energy Mater., 2014, 4, 1301659.

(26) Brinkmann, M. ; Hartmann, L. ; Biniek, L. ; Tremel, K. and Kayunkid, N. Macromol. Rapid. Comm. 2014, 35, 9.

(27) Hamidi-Sakr, A.; Biniek, L.; Fall, S.; Brinkmann, M. Adv. Funct. Mater. 2016, 26, $408-420$.

(28) Zhang, S.; Yang, B.; Liu, D.; Zhang, H.; Zhao, W.; Wang, Q.; He, C.; Hou, J. Macromolecules 2016, 49, 120-126.

(29) Ibraikulov, O. A.; Heinrich, B.; Leclerc, N.; Chávez, P.; Bulut, I.; Ngov, C.; Boyron, O.; Brouckaert, N.; Swaraj, S.; Gerasimov, K. L.; Ivanov, D. A.; Mery, S.; Lévêque, P. and Heiser, T. submitted.

(30) Brinkmann, M.; Rannou, P. Macromolecules 2009, 42, 1125-1130. 
(31) Kayunkid, N.; Uttiya, S.; Brinkmann, M. Macromolecules 2010, 43, 4961-4967.

(32) Biniek, L. ; Hamidi-Sakr, A. ; Grodd, L. ; Escoubas, S. ; Dappe, Y. J. ; Grigorian, S. ; Schmutz, M. ; Brinkmann, M. Adv. Electron. Mater. 2018, 1700480.

(33) Nielsen, C. B. , White, A. J. P. and McCulloch, I. J. Organ. Chem. 2015, 80, 5045

(34) O’Connor, B.; Kline, R. J.; Conrad, B. R.; Richter, L. J.; Gundlach, D.; Toney, M. F. and DeLongchamp, D. M. Adv. Funct. Mat. 2011, 21, 3697.

(35) Luzio, A.; Criante, L.; D’Innocenzo, V.; Caironi, M. Sci. Rep. 2013, 3, 3425.

(36) B.-G. Kim, E.-J. Jeong, J. W. Chung, S. Seo, B. Koo and J. Kim, Nat. Mat. 2013, 12, 659.

(37) Patel, S. N.; Su, G. M.; Luo, C.; Wang, M.; Perez, L. A.; Fischer, D. A.; Prendergast, D.; Bazan, G. C.; Heeger, A. J.; Chabinyc, M. L.; Kramer, E. J. Macromolecules 2015, 48, 6606-6616.

(38) Hamidi-Sakr, A.; Schiefer, D.; Covindarassou, S.; Biniek, L.; Sommer, M.; Brinkmann, M. Macromolecules 2016, 49, 3452-3462

(39) Mandelkern, L. in Crystallization of Polymers, volume 2, Kinetics and Mechanisms, Cambridge University Press, UK, 2004.

(40) Causin, V.; Marega, C.; Margio, A.; Valentini, L. and Kenny, J. M. Macromolecules 2005, 38, 409.

(41) Chang, S. W.; Waters, H.; Kettle, J. and Horie, M. Org. Electr. 2012, 13, 2967.

(42) Lotz, B.; Lovinger, A. J. and Cais, R. E. Macromolecules 1988, 21, 2375. 
(43) Lovinger, A. J.; Lotz, B. , Davis, D. D. and Padden, F. J. Macromolecules 1993, 26, 3494.

(44) Schulz, G. L.; Fischer, F. S. U.; Trefz, D.; Melnyk, A.; Hamidi-Sakr, A.; Brinkmann, M.; Andrienko, D.; Ludwigs, S. Macromolecules 2017, 50, 1402-1414. 


\section{Segregated versus disordered stacking in two low bandgap alternated copolymers for photovoltaic applications : Impact of polymorphism on optical properties}

Yuhan Zhong ${ }^{1}$, Laure Biniek ${ }^{1}$, Nicolas Leclerc ${ }^{2}$, Stéphanie Ferry² and

Martin Brinkmann ${ }^{1}$

(1) Institut Charles Sadron, CNRS-Université de Strasbourg, 23 rue du Loess, Strasbourg 67034, France

(2) Institut de Chimie et Procédés pour l'Energie, l’Environnement et la Santé, UMR 7515, ECPM, 25 rue Becquerel, 67087 Strasbourg Cedex2, France 
1) Synthesis of PDTBT-TT.

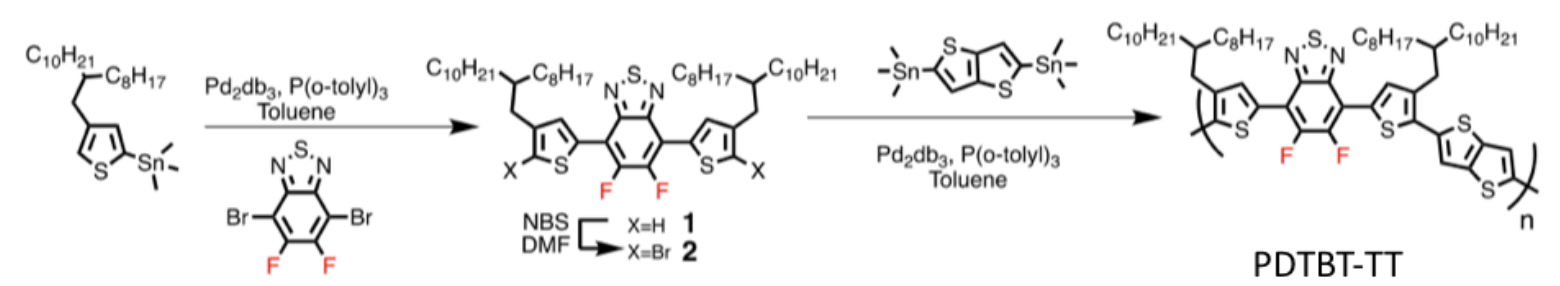

Figure S1 : Synthesis route of PDTBT-TT.

\section{5,6-difluoro-4,7-bis(4-(2-octyldodecyl)thiophen-2-yl)benzo[c][1,2,5]thiadiazole (compound 1).}

A flame dried Schlenck was charged with the 4,7-Dibromo-5,6-difluoro-2,1,3benzothiadiazole (1.0 equiv) and the 2-(trimethylstannyl)-4-(2-octyldodecyl))thiophene compound (2.2 equiv). Anhydrous and degassed toluene (0.1M) was added under inert gas. Finally, the Pd2(dba)3 (2.0 mol\%) and P(o-tolyl)3 (8.0 mol\%) were added in one portion and the mixture was stirred 24 hours at $120^{\circ} \mathrm{C}$. After cooling to room temperature the reaction mixture was filtered through a pad of celite and the toluene solution was evaporated under reduced pressure. Then, the crude material was purified by column chromatography. The crude product was purified by column chromatography (silica gel, petroleum ether). Yield: 65\%.

${ }^{1} \mathrm{H} \mathrm{NMR}\left(400 \mathrm{MHz}, \mathrm{CDCl}_{3}\right) \delta(\mathrm{ppm})=8.09\left(\mathrm{~d},{ }^{4} J=1.1 \mathrm{~Hz}, 2 \mathrm{H}\right), 7.17\left(\mathrm{~d},{ }^{4} J=1.0 \mathrm{~Hz}, 2 \mathrm{H}\right), 2.65$ $\left(\mathrm{d},{ }^{3} \mathrm{~J}=6.7 \mathrm{~Hz}, 4 \mathrm{H}\right), 1.70(\mathrm{~m}, 2 \mathrm{H}), 1.26(\mathrm{~m}, 64 \mathrm{H}), 0.87(\mathrm{t}, 3 \mathrm{~J}=6.0 \mathrm{~Hz}, 12 \mathrm{H})$.

${ }^{13} \mathrm{C} \mathrm{NMR}\left(100 \mathrm{MHz}, \mathrm{CDCl}_{3}\right) \delta(\mathrm{ppm})=151.4(\mathrm{dd}, J=256.5$ and $20.4 \mathrm{~Hz}), 149.0(\mathrm{t}, J=4.23$ $\mathrm{Hz}), 142.37,132.9$ (d, J=3.8 Hz), 131.0, 124.81, 111.69 (dd, J=9.1 and 4.9 Hz), 39.0, 34.9, $33.4,31.9,30.0,29.7,29.6,29.3,26.7,22.6,14.0$. thiadiazole (compound 2) 
The compound 1 (1.0 equiv) was solubilized in DMF (0.05 M) under argon in the dark. NBS (2.0 equiv) was added portion wise. The resulting solution was stirred at room temperature under argon overnight. Water and diethylether were added and the resulting solution was stirred for $2 \mathrm{~h}$. The organic phase was separated from the water phase and extracted with brine ( $3 \times 100 \mathrm{~mL})$. The organic phase was dried with sodium sulfate, filtered and the solvent evaporated under reduced pressure. The crude product was purified by column chromatography. The crude product was purified by column chromatography (silica gel, petroleum ether). Yield: 78\%.

${ }^{1} \mathrm{H} \mathrm{NMR}\left(400 \mathrm{MHz}, \mathrm{CDCl}_{3}\right) \delta(\mathrm{ppm})=7.90(\mathrm{~s}, 2 \mathrm{H}), 2.57(\mathrm{~d}, 3 \mathrm{~J}=7.0 \mathrm{~Hz}, 4 \mathrm{H}), 1.74(\mathrm{~m}, 2 \mathrm{H})$, $1.25(\mathrm{~m}, 64 \mathrm{H}), 0.86(\mathrm{t}, 3 \mathrm{~J}=5.4 \mathrm{~Hz}, 12 \mathrm{H})$.

${ }^{13} \mathrm{C} \mathrm{NMR}\left(100 \mathrm{MHz}, \mathrm{CDCl}_{3}\right) \delta(\mathrm{ppm})=152.4(\mathrm{dd}, J=260.3$ and $20.4 \mathrm{~Hz}), 148.4(\mathrm{t}, J=4.2$ $\mathrm{Hz}), 141.8,132.3(\mathrm{t}, J=4.7 \mathrm{~Hz}), 131.0,115.1$ (t, J= 3.6 Hz), $111.0(\mathrm{dd}, J=4.7$ and $13.3 \mathrm{~Hz}$ ), 38.6, 34.2, 33.4, 31.9, 30.0, 29.7, 29.65, 29.61, 29.3, 26.6, 22.7, 14.1 .

\section{PDTBT-TT, Stille polymerization.}

A flame dried Schlenck was charged with dibrominated compound 2 (1.0 equiv) and the 2-5-bis-trimethylstannyl-thieno[3,2-b]thiophene (1.0 equiv). Anhydrous and degassed toluene $(0.0125 \mathrm{M})$ was added under inert gas. Then, the $\mathrm{Pd}_{2}(\mathrm{dba})_{3}(2 \mathrm{~mol} \%)$ and $\mathrm{P}(\mathrm{o}-$ tolyl $)_{3}(8 \mathrm{~mol} \%)$ were added in one portion and the mixture was stirred 24 hours at $120^{\circ} \mathrm{C}$. The reaction was quenched with 2 -(trimethylstannyl)-thiophene (0.6 equiv) during 1 hour followed by 2-bromothiophene ( 0.6 equiv). Then, the polymer crude was purified by precipitation in methanol, filtered and separated by Soxhlet extraction with methanol, acetone, cyclohexane and chlorobenzene. Then, the sodium diethyldithiocarbamate solution was added in the chlorobenzene fraction and the mixture was stirred at $60^{\circ} \mathrm{C}$ during 1 hour. The organic phase was washed with water, separated and evaporated under reduced pressure. Finally, the polymer was precipitated in methanol, filtered and dried under reduced pressure at $40^{\circ} \mathrm{C}$ overnight, providing powder with a metallic shine. 
Figure S2. Dependence of the dichroic ratio of the 0-0 component at $697 \mathrm{~nm}$ versus rubbing temperature $T_{R}$ for PDTBT-TT.

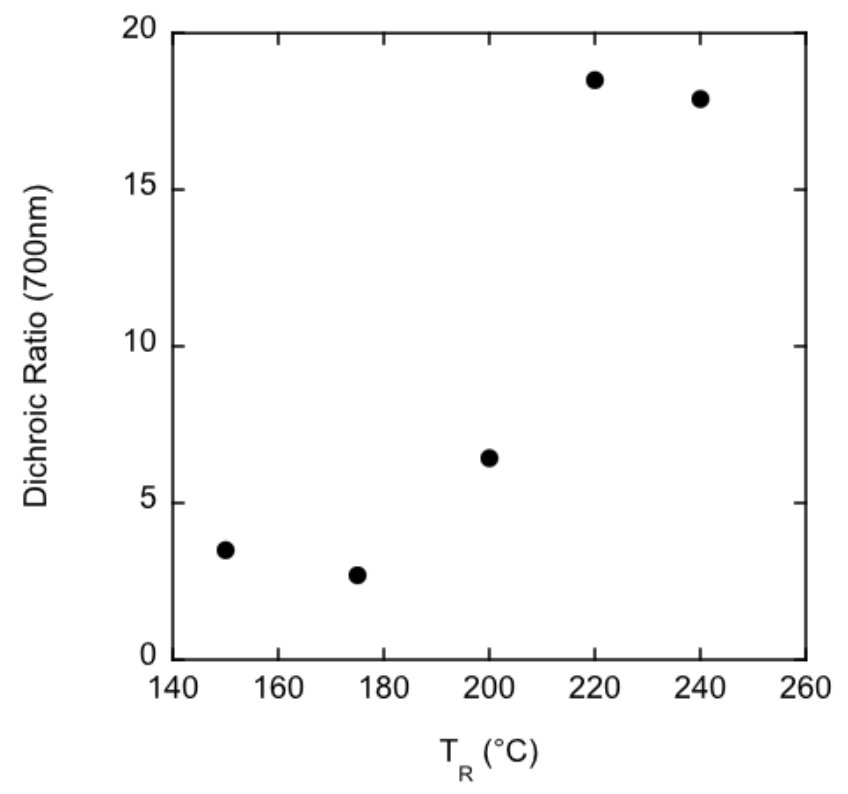



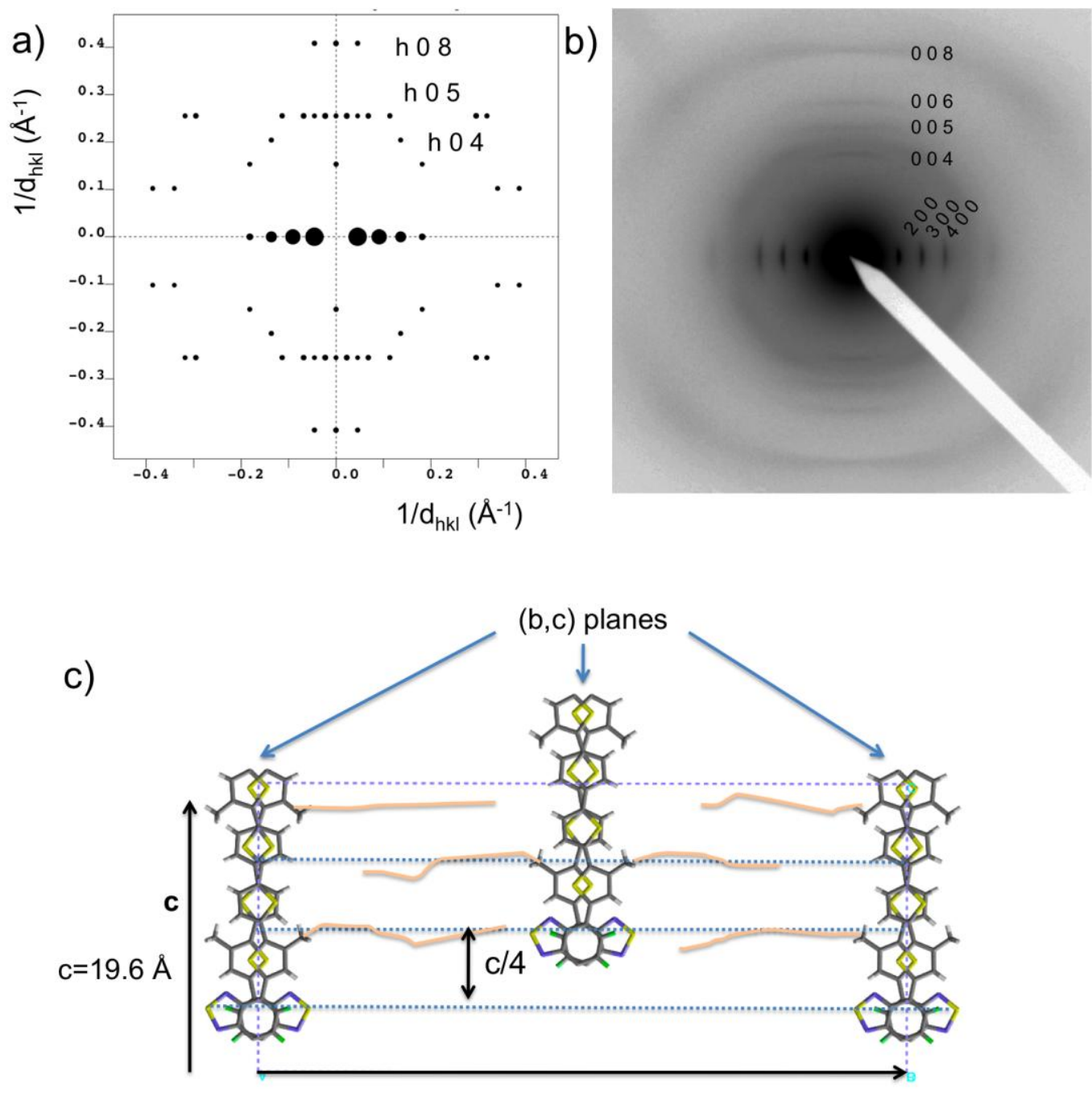

$a=2 \times 22.2 \AA$

Figure S3. Comparison of the calculated (a) and experimental ED pattern for the [0 10 l 0 ] zone of PBT4T. (c) (010) projection of the refined model corresponding to a segregated stacking of the chains. Disordered side chains are schematically drawn in pink. The unit cell is doubled along the a axis to take into account the $c / 4$ shift between successive $(b, c)$ planes of $\pi$-stacked chains that is required for a good interdigitation of side chains. In the true structure, the shift between successive $(b, c)$ planes is statistical in essence and it is given by $\pm(2 n+1) c / 4$ ( $n$ is an integer). Note that the intensity of $h 04$ reflections is underestimated as the alkyl side chains are not taken into account for the calculation of the ED pattern. Including such side chains would strongly enhance the h 04 reflections. 